\title{
Fluorine-18-fluorodeoxyglucose (FDG) positron emission tomography (PET) computed tomography (CT) for the detection of bone, lung, and lymph node metastases in rhabdomyosarcoma
}

\author{
Vaarwerk, Bas ; Breunis, Willemijn B ; Haveman, Lianne M ; de Keizer, Bart ; Jehanno, Nina ; \\ Borgwardt, Lise ; van Rijn, Rick R ; van den Berg, Henk ; Cohen, Jérémie F ; van Dalen, Elvira C ; \\ Merks, Johannes H M
}

\begin{abstract}
Rhabdomyosarcoma (RMS) is the most common paediatric soft-tissue sarcoma and can emerge throughout the whole body. For patients with newly diagnosed RMS, prognosis for survival depends on multiple factors such as histology, tumour site, and extent of the disease. Patients with metastatic disease at diagnosis have impaired prognosis compared to those with localised disease. Appropriate staging at diagnosis therefore plays an important role in choosing the right treatment regimen for an individual patient. Fluorine-18-fluorodeoxyglucose (18F-FDG) positron emission tomography (PET) is a functional molecular imaging technique that uses the increased glycolysis of cancer cells to visualise both structural information and metabolic activity. 18F-FDG-PET combined with computed tomography (CT) could help to accurately stage the extent of disease in patients with newly diagnosed RMS. In this review we aimed to evaluate whether $18 \mathrm{~F}-\mathrm{FDG}$-PET could replace other imaging modalities for the staging of distant metastases in RMS. Objectives To determine the diagnostic accuracy of 18F-FDG-PET/CT imaging for the detection of bone, lung, and lymph node metastases in RMS patients at first diagnosis. Search methods We searched MEDLINE in PubMed (from 1966 to 23 December 2020) and Embase in Ovid (from 1980 to 23 December 2020) for potentially relevant studies. We also checked the reference lists of relevant studies and review articles; scanned conference proceedings; and contacted the authors of included studies and other experts in the field of RMS for information about any ongoing or unpublished studies. We did not impose any language restrictions. Selection criteria We included cross-sectional studies involving patients with newly diagnosed proven RMS, either prospective or retrospective, if they reported the diagnostic accuracy of 18F-FDG-PET/CT in diagnosing lymph node involvement or bone metastases or lung metastases or a combination of these metastases. We included studies that compared the results of the 18 F-FDG-PET/CT imaging with those of histology or with evaluation by a multidisciplinary tumour board as reference standard. Data collection and analysis Two review authors independently performed study selection, data extraction, and methodological quality assessement according to Quality Assessment of Diagnostic Accuracy Studies 2 (QUADAS-2). We analysed data for the three outcomes (nodal involvement and lung and bone metastases) separately. We used data from the $2 \times 2$ tables (consisting of true positives, false positives, true negatives, and false negatives) to calculate sensitivity and specificity in each study and corresponding 95\% confidence intervals. We did not consider a formal meta-analysis to be relevant because of the small number of studies and substantial heterogeneity between studies. Main results Two studies met our inclusion criteria. The diagnostic accuracy of 18F-FDG-PET/CT was reported in both studies, which included a total of 36 participants. We considered both studies to be at high risk of bias for the domain reference standard. We considered one study to be at high risk of bias for the domain index test and flow and timing. Sensitivity and specificity of 18F-FDG-PET/CT for the detection of bone metastases was $100 \%$ in both studies (95\% confidence interval (CI) for sensitivity was $29 \%$ to $100 \%$ in study one and $40 \%$ to $100 \%$ in study two; $95 \%$ CI for specificity was $83 \%$ to $100 \%$ in study one and $66 \%$ to $100 \%$ in study two). The reported sensitivity of $18 \mathrm{~F}-\mathrm{FDG}-\mathrm{PET} / \mathrm{CT}$ for the detection of lung metastases was not calculated since only two participants in study two showed lung
\end{abstract}


metastases, of which one was detected by 18 F-FDG-PET/CT. Reported specificity was $96 \%$ in study one (95\% CI $78 \%$ to $100 \%)$ and $100 \%$ (95\% CI $72 \%$ to $100 \%)$ in study two. The reported sensitivity for the detection of nodal involvement was $100 \%$ (95\% CI $63 \%$ to $100 \%$ in study one and $40 \%$ to $100 \%$ in study two); the reported specificity was $100 \%$ (95\% CI $78 \%$ to 100\%) in study one and $89 \%$ (95\% CI $52 \%$ to $100 \%)$ in study two.

DOI: https://doi.org/10.1002/14651858.CD012325.pub2

Posted at the Zurich Open Repository and Archive, University of Zurich ZORA URL: https://doi.org/10.5167/uzh-212928

Journal Article

Published Version

Originally published at:

Vaarwerk, Bas; Breunis, Willemijn B; Haveman, Lianne M; de Keizer, Bart; Jehanno, Nina; Borgwardt, Lise; van Rijn, Rick R; van den Berg, Henk; Cohen, Jérémie F; van Dalen, Elvira C; Merks, Johannes H M (2021). Fluorine-18-fluorodeoxyglucose (FDG) positron emission tomography (PET) computed tomography (CT) for the detection of bone, lung, and lymph node metastases in rhabdomyosarcoma. Cochrane Database of Systematic Reviews, 11:CD012325.

DOI: https://doi.org/10.1002/14651858.CD012325.pub2 


\section{Fluorine-18-fluorodeoxyglucose (FDG) positron emission tomography (PET) computed tomography (CT) for the detection of bone, lung, and lymph node metastases in rhabdomyosarcoma} (Review)

Vaarwerk B, Breunis WB, Haveman LM, de Keizer B, Jehanno N, Borgwardt L, van Rijn RR, van den Berg H, Cohen JF, van Dalen EC, Merks JHM

Vaarwerk B, Breunis WB, Haveman LM, de Keizer B, Jehanno N, Borgwardt L, van Rijn RR, van den Berg H, Cohen JF, van Dalen EC, Merks JHM.

Fluorine-18-fluorodeoxyglucose (FDG) positron emission tomography (PET) computed tomography (CT) for the detection of bone, lung, and lymph node metastases in rhabdomyosarcoma.

Cochrane Database of Systematic Reviews 2021, Issue 11. Art. No.: CD012325.

DOI: 10.1002/14651858.CD012325.pub2.

www.cochranelibrary.com

Fluorine-18-fluorodeoxyglucose (FDG) positron emission tomography (PET) computed tomography (CT) for the detection of bone, lung, and lymph node metastases in rhabdomyosarcoma (Review)

Copyright (c) 2021 The Cochrane Collaboration. Published by John Wiley \& Sons, Ltd. 
TABLE OF CONTENTS

ABSTRACT 1

PLAIN LANGUAGE SUMMARY

SUMMARY OF FINDINGS

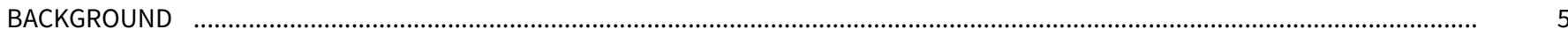

OBJECTIVES

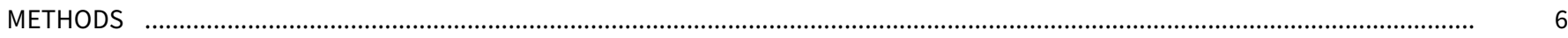

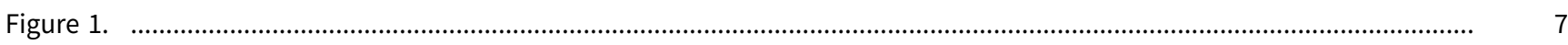

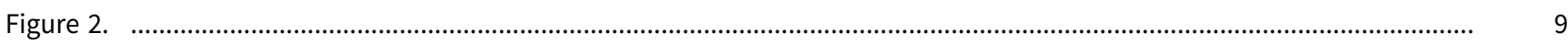

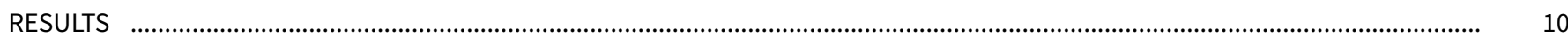

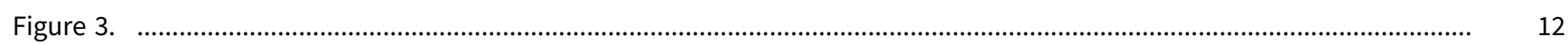

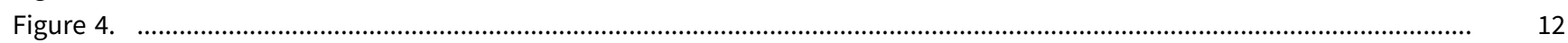

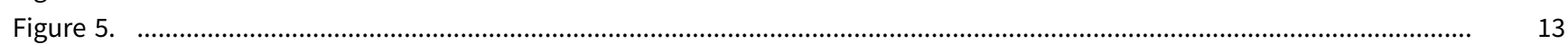

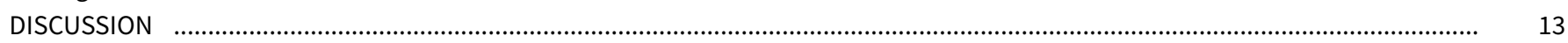

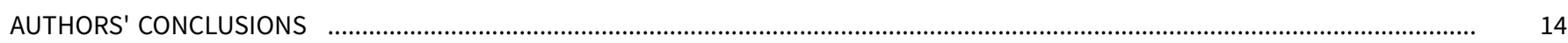

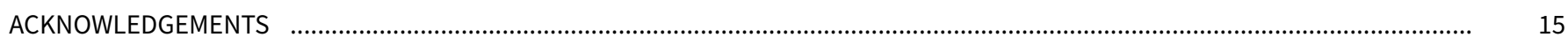

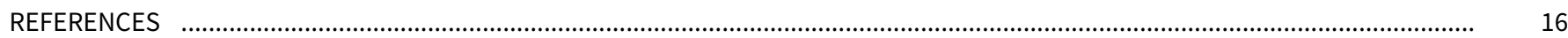

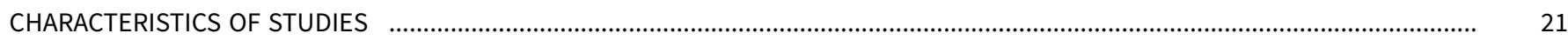

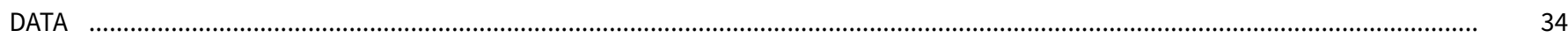

Test 1. 18F-FDG-PET/CT for detection of bone metastases

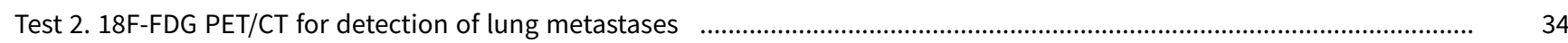

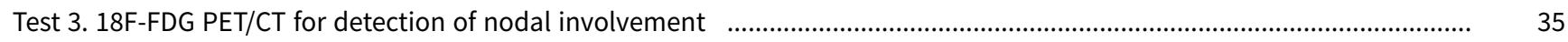

ADDITIONAL TABLES

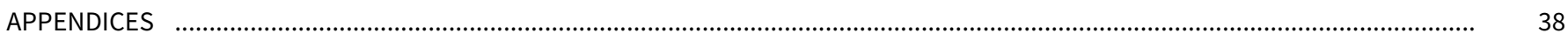

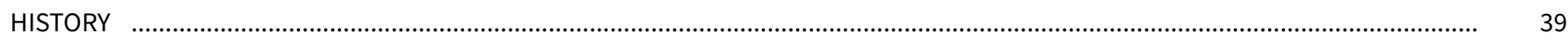

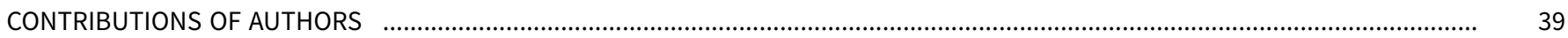

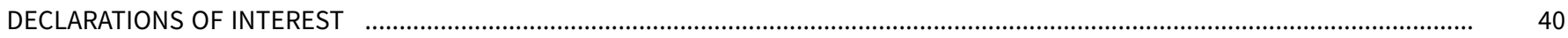

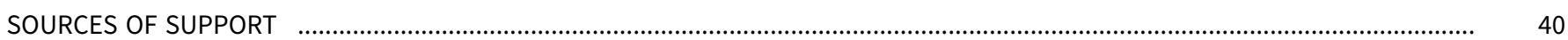

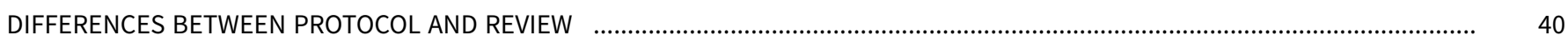

INDEX TERMS

Fluorine-18-fluorodeoxyglucose (FDG) positron emission tomography (PET) computed tomography (CT) for the detection of bone, lung,

Copyright $\odot 2021$ The Cochrane Collaboration. Published by John Wiley \& Sons, Ltd. 
[Diagnostic Test Accuracy Review]

\section{Fluorine-18-fluorodeoxyglucose (FDG) positron emission tomography (PET) computed tomography (CT) for the detection of bone, lung, and lymph node metastases in rhabdomyosarcoma}

Bas Vaarwerk1,2, Willemijn B Breunis2,3, Lianne M Haveman1,2, Bart de Keizer ${ }^{1}$, Nina Jehanno ${ }^{4}$, Lise Borgwardt ${ }^{5}$, Rick R van Rijn6 ${ }^{6}$, Henk van den Berg ${ }^{2}$, Jérémie F Cohen ${ }^{7,8}$, Elvira C van Dalen ${ }^{1}$, Johannes HM Merks ${ }^{1,2}$

1Princess Máxima Center for Pediatric Oncology, Utrecht, Netherlands. 2Department of Paediatric Oncology, Emma Children's Hospital, Amsterdam UMC, University of Amsterdam, Amsterdam, Netherlands. 3Department of Oncology and Children's Research Center, University Children's Hospital Zurich, Zurich, Switzerland. ${ }^{4}$ Department of Nuclear Medicine, Institut Curie, Paris, France. ${ }^{5}$ Department of Clinical Physiology, Nuclear Medicine and PET, Rigshospitalet, Copenhagen University Hospital, Copenhagen, Denmark. ${ }^{6}$ Department of Radiology and Nuclear Medicine, Amsterdam UMC, University of Amsterdam, Amsterdam, Netherlands. ${ }^{7}$ Obstetrical, Perinatal and Pediatric Epidemiology Research Team (EPOPé), Centre of Research in Epidemiology and Statistics (CRESS), UMR1153, Université de Paris, Paris, France. ${ }^{8}$ Department of General Pediatrics and Pediatric Infectious Diseases, Necker - Enfants malades hospital, Assistance Publique - Hôpitaux de Paris, Paris Descartes University, Paris, France

Contact: Johannes HM Merks, j.h.m.merks@prinsesmaximacentrum.nl.

Editorial group: Cochrane Childhood Cancer Group.

Publication status and date: New, published in Issue 11, 2021.

Citation: Vaarwerk B, Breunis WB, Haveman LM, de Keizer B, Jehanno N, Borgwardt L, van Rijn RR, van den Berg H, Cohen JF, van Dalen EC, Merks JHM. Fluorine-18-fluorodeoxyglucose (FDG) positron emission tomography (PET) computed tomography (CT) for the detection of bone, lung, and lymph node metastases in rhabdomyosarcoma. Cochrane Database of Systematic Reviews 2021, Issue 11. Art. No.: CD012325. DOI: 10.1002/14651858.CD012325.pub2.

Copyright (c 2021 The Cochrane Collaboration. Published by John Wiley \& Sons, Ltd.

\section{A B S T R A C T}

\section{Background}

Rhabdomyosarcoma (RMS) is the most common paediatric soft-tissue sarcoma and can emerge throughout the whole body. For patients with newly diagnosed RMS, prognosis for survival depends on multiple factors such as histology, tumour site, and extent of the disease. Patients with metastatic disease at diagnosis have impaired prognosis compared to those with localised disease. Appropriate staging at diagnosis therefore plays an important role in choosing the right treatment regimen for an individual patient.

Fluorine-18-fluorodeoxyglucose (18F-FDG) positron emission tomography (PET) is a functional molecular imaging technique that uses the increased glycolysis of cancer cells to visualise both structural information and metabolic activity. ${ }^{18}$ F-FDG-PET combined with computed tomography (CT) could help to accurately stage the extent of disease in patients with newly diagnosed RMS. In this review we aimed to evaluate whether 18 F-FDG-PET could replace other imaging modalities for the staging of distant metastases in RMS.

\section{Objectives}

To determine the diagnostic accuracy of 18F-FDG-PET/CT imaging for the detection of bone, lung, and lymph node metastases in RMS patients at first diagnosis.

\section{Search methods}

We searched MEDLINE in PubMed (from 1966 to 23 December 2020) and Embase in Ovid (from 1980 to 23 December 2020) for potentially relevant studies. We also checked the reference lists of relevant studies and review articles; scanned conference proceedings; and contacted the authors of included studies and other experts in the field of RMS for information about any ongoing or unpublished studies. We did not impose any language restrictions. 


\section{Selection criteria}

We included cross-sectional studies involving patients with newly diagnosed proven RMS, either prospective or retrospective, if they reported the diagnostic accuracy of 18 F-FDG-PET/CT in diagnosing lymph node involvement or bone metastases or lung metastases or a combination of these metastases. We included studies that compared the results of the 18F-FDG-PET/CT imaging with those of histology or with evaluation by a multidisciplinary tumour board as reference standard.

\section{Data collection and analysis}

Two review authors independently performed study selection, data extraction, and methodological quality assessement according to Quality Assessment of Diagnostic Accuracy Studies 2 (QUADAS-2). We analysed data for the three outcomes (nodal involvement and lung and bone metastases) separately. We used data from the $2 \times 2$ tables (consisting of true positives, false positives, true negatives, and false negatives) to calculate sensitivity and specificity in each study and corresponding $95 \%$ confidence intervals. We did not consider a formal meta-analysis to be relevant because of the small number of studies and substantial heterogeneity between studies.

\section{Main results}

Two studies met our inclusion criteria. The diagnostic accuracy of $18 \mathrm{~F}-\mathrm{FDG}$-PET/CT was reported in both studies, which included a total of 36 participants. We considered both studies to be at high risk of bias for the domain reference standard. We considered one study to be at high risk of bias for the domain index test and flow and timing. Sensitivity and specificity of $18 \mathrm{~F}-\mathrm{FDG}$-PET/CT for the detection of bone metastases was $100 \%$ in both studies ( $95 \%$ confidence interval (Cl) for sensitivity was $29 \%$ to $100 \%$ in study one and $40 \%$ to $100 \%$ in study two; $95 \% \mathrm{Cl}$ for specificity was $83 \%$ to $100 \%$ in study one and $66 \%$ to $100 \%$ in study two). The reported sensitivity of $18 \mathrm{~F}-\mathrm{FDG}$-PET/CT for the detection of lung metastases was not calculated since only two participants in study two showed lung metastases, of which one was detected by 18 F-FDG-PET/CT. Reported specificity was $96 \%$ in study one $(95 \% \mathrm{Cl} 78 \%$ to $100 \%)$ and $100 \%(95 \% \mathrm{Cl} 72 \%$ to $100 \%)$ in study two. The reported sensitivity for the detection of nodal involvement was $100 \%$ (95\% Cl $63 \%$ to $100 \%$ in study one and $40 \%$ to $100 \%$ in study two); the reported specificity was $100 \%(95 \% \mathrm{Cl} 78 \%$ to $100 \%)$ in study one and $89 \%(95 \% \mathrm{Cl} 52 \%$ to $100 \%)$ in study two.

\section{Authors' conclusions}

The diagnostic accuracy of 18 F-FDG-PET/CT for the detection of bone, lung, and lymph node metastases was reported in only two studies including a total of only 36 participants with newly diagnosed RMS. Because of the small number of studies (and participants), there is currently insufficient evidence to reliably determine the diagnostic accuracy of 18F-FDG-PET/CT in the detection of distant metastases. Larger series evaluating the diagnostic accuracy of 18 F-FDG-PET/CT for the detection of metastases in patients with RMS are necessary.

\section{PLAIN LANGUAGE SUMMARY}

\section{The accuracy of ${ }^{18}$ F-FDG-PET/CT for the detection of metastatic rhabdomyosarcoma in newly diagnosed patients}

\section{Why is accurate staging of rhabdomyosarcoma important?}

Rhabdomyosarcoma (RMS) accounts for $3 \%$ to $5 \%$ of all childhood cancers. Treatment consists of multidrug chemotherapy and surgery and/or radiotherapy. Treatment for newly diagnosed patients depends on the extent of the cancer. Five-year survival for patients with localised disease is around $75 \%$, whereas it is below $30 \%$ for patients with disease that has spread to different part(s) of the body (metastatic disease). Survival for metastatic patients depends on the number of metastases as well as the site(s) of metastases. It is of great importance to accurately stage the extent of the disease because not recognising patients with metastatic disease would lead to undertreatment, whereas incorrectly identifying lesions as being metastatic would lead to overtreatment. ${ }^{18} \mathrm{~F}-\mathrm{FDG}$-PET/CT is a special imaging technique that could be helpful in visualising the extent of the disease in patients with newly diagnosed RMS. However, the accuracy (the ability to separate RMS metastases from other lesions) of 18 F-FDG-PET/CT is currently unknown.

\section{What was the aim of this review?}

We wanted to find out how accurate 18F-FDG-PET/CT is for the detection of bone and lung metastases and lymph node involvement in patients with newly diagnosed RMS.

\section{What was studied in this review?}

We searched scientific literature databases for studies comparing the results of 18F-FDG-PET/CT to standard staging evaluation (histologic examinations (using a microscope to examine tissues and cells) or results of other imaging methods discussed by a multidisciplinary tumour board). The advantage of using 18F-FDG-PET/CT compared to standard staging evaluations would be the use of 18 F-FDG-PET/CT as a single diagnostic test to detect metastases, thus reducing patient burden and lowering radiation exposure.

\section{Main results}

We included two studies involving a total of 36 participants with RMS. Because of the low number of participants in the included studies and the differences in quality between studies, we were not able to calculate summary values of sensitivity (the proportion of patients who 
have the condition and who received a positive result on the test) and specificity (the proportion of patients who do not have the condition and who received a negative result on the test), and the reported results should be viewed with caution.

The two included studies reported a sensitivity and specificity of $100 \%$ of 18 F-FDG-PET/CT for the detection of bone metastases. The sensitivity for the detection of lung metastases was $50 \%$ in one study, and could not be estimated in the other study; specificity ranged from $96 \%$ to $100 \%$. In both studies, the sensitivity for the detection of lymph node involvement was $100 \%$, and specificity ranged from $89 \%$ to $100 \%$.

\section{How reliable are the results of the studies in this review?}

The main limitation of this review was that the findings were based on only two studies with a total of 36 participants and due to the design of the studies we considered them to be at high risk of bias on specific domains.

In the included studies, histopathological (microscopic examination of tissue) confirmation was considered the optimal reference standard (the best available method to determine whether the condition is present or absent) to determine RMS metastases from other lesions; however, this was not done in all participants. In cases where no histopathological confirmation was done, the judgement of a multidisciplinary tumour board was considered the reference standard. Judgement of the multidisciplinary tumour boards was partly based on 18 F-FDG-PET/CT imaging results, potentially resulting in overestimation of sensitivity and specificity. Furthermore, in one included study the participants all underwent the same diagnostic procedures, whereas in the other study this was not the case for all participants; this study did not clearly define what was considered a positive test result for 18F-FDG-PET/CT imaging. This might have biased the results.

\section{What are the implications of this review?}

The total number of studies and participants was too low to allow us to draw any firm conclusions. Large studies evaluating the accuracy of $18 \mathrm{~F}-\mathrm{FDG}-\mathrm{PET} / \mathrm{CT}$ in patients with RMS are needed.

\section{How up-to-date is this review?}

We searched for and used studies published from 1966 to 23 December 2020. 


\section{SUMMARY OF FINDINGS}

\section{Summary of findings 1 . Objective: diagnostic accuracy of 18 F-FDG-PET/CT for the detection of bone and lung metastases and lymph node involvement in newly diagnosed rhabdomyosarcoma}

\begin{tabular}{|c|c|c|c|}
\hline $\begin{array}{l}\text { Patients/popula- } \\
\text { tion: }\end{array}$ & \multicolumn{3}{|c|}{ Patients with histology proven RMS at first diagnosis } \\
\hline Index test: & \multicolumn{3}{|c|}{ 18F-FDG-PET/CT } \\
\hline $\begin{array}{l}\text { Reference stan- } \\
\text { dard: }\end{array}$ & \multicolumn{3}{|c|}{$\begin{array}{l}\text { Biopsy with histological examination of all suspected lesions, or if not available judgement of a multidiscipli- } \\
\text { nary tumour board based on: } \\
\text { clinical findings, results of conventional imaging (i.e. whole-body } 99 \mathrm{mT} \text { T skeleton scintigraphy, chest CT scan, } \\
\text { X-ray thorax, MRI, ultrasound), histology of selected lesions, follow-up }\end{array}$} \\
\hline Studies: & \multicolumn{3}{|c|}{ Retrospective cross-sectional studies, 2 in total (number of participants enrolled: 36 ) } \\
\hline Subgroup & Sensitivity & Specificity & $\begin{array}{l}\text { Number of patients with event/total } \\
\text { number of participants }\end{array}$ \\
\hline Bone metastases & $100 \%$ & $100 \%$ & $7 / 36$ \\
\hline Lung metastases & $50 \% ¥$ & Range: $96 \%$ to $100 \%$ & $2 / 36$ \\
\hline $\begin{array}{l}\text { Nodal involve- } \\
\text { ment }\end{array}$ & $100 \%$ & Range: $89 \%$ to $100 \%$ & $12 / 36$ \\
\hline
\end{tabular}

¥Sensitivity of lung metastases only reported in Ricard 2011. No patients in Eugene 2012 had lung metastases.

Abbreviations: 18F-FDG-PET/CT: fluorine-18-fluorodeoxyglucose - positron emission tomography/computed tomography; $99 \mathrm{mTC}$ bone scintigraphy: technetium-99m bone scintigraphy; CT: computed tomography; MRI: magnetic resonance imaging; RMS: rhabdomyosarcoma. 


\section{B A C K G R O U N D}

\section{Target condition being diagnosed}

Rhabdomyosarcoma (RMS) is the most common paediatric softtissue sarcoma and constitutes about $3 \%$ to $5 \%$ of all malignancies in childhood (Miller 1995; Ward 2014). The annual incidence in children varies between four per million and seven per million depending on the age group. About 340 new cases are diagnosed in children each year in the USA (Ward 2014). RMS is a tumour of mesenchymal cell origin and can arise throughout the whole body. About $40 \%$ of RMS arises in the head and neck area, $25 \%$ to $30 \%$ in the genito-urinary region, $15 \%$ in the extremities, and $15 \%$ to $20 \%$ in other regions (e.g. trunk) (McDowell 2003; Weiss 2013). Prognosis for patients with localised disease is based on several factors including histology, tumour site and size, postsurgical stage (Intergroup Rhabdomyosarcoma Studies (IRS) grouping), nodal status, distant metastasis, and patient's age. In children, two main histological subtypes have been identified: embryonal (ERMS) and alveolar (ARMS). The prognosis of patients with ARMS is significantly worse compared to patients with ERMS (Meza 2006). Orbital site, head and neck non-parameningeal, and genito-urinary non-bladder/prostate sites have favourable prognosis compared to other sites. Younger patients (aged less than 10 years) and patients with small tumours (less than $5 \mathrm{~cm}$ ) do better than older patients or patients with large tumours. Patients with completely resected tumours do better than those with residual disease. In about $21 \%$ of RMS patients lymph nodes are involved (Weiss 2013), negatively influencing prognosis. Distant metastases are identified in about $16 \%$ of newly diagnosed RMS patients (including $6 \%$ lung metastases and $5 \%$ bone metastases). Prognosis for patients with metastatic RMS compares unfavourably to patients with localised disease, and prognostic factors for patients with metastatic tumours include age, primary tumour site (patients with extremity and other sites have dismal prognosis), presence of bone or bone marrow metastases, and number of metastatic sites (Oberlin 2008). RMS patients are subdivided into risk groups based on these risk factors (Arndt 2009; Arndt 2013; Meza 2006; Pappo 2007; Raney 2001; Raney 2011). In current treatment protocols, intensity of chemotherapy and application of radiotherapy to the primary site, involved nodes, and metastatic sites is tailored based on these risk categories (EpSSG RMS 2005 (Bisogno 2018; NCT00379457), COG ARST0531 (NCT00354835)). With current multimodal treatment protocols, five-year overall survival for RMS patients is about 65\% (Gatta 2014; Ward 2014). However, survival for patients differs dramatically between those with and those without metastasis. Patients with local disease at diagnosis have a five-year overall survival of around $75 \%$, whereas this is below $30 \%$ in metastatic RMS patients (Crist 2001; Oberlin 2008). Patients with lung metastases have a better outcome than those with bone or bone marrow metastases. Moreover, patients with more than two metastatic sites have a more dismal outcome compared to those with only one metastatic site (Oberlin 2008). To apply the most optimal treatment in terms of survival but also in term of late effects, risk group stratification for individual patients at diagnosis is extremely important.

\section{Index test(s)}

Fluorine-18-fluorodeoxyglucose (FDG) positron emission tomography (PET) is a functional molecular imaging technique that uses the increased glycolysis of cancer cells to visualise both structural information and metabolic activity. By combining ${ }^{18} \mathrm{~F}-$
FDG-PET with computed tomography (CT), the exact anatomical location and structural information of the lesion can be acquired (Gambhir 2002). In several cancer types, such as lung cancer and lymphoma, FDG- PET/CT has proven to be of important value in accurately staging at diagnosis (Gallamini 2014). ${ }^{18} \mathrm{~F}$ FDG-PET/CT is being evaluated for clinical use in patients with sarcoma (Quak 2011). Several studies on children with sarcoma report the additional value of using $18 \mathrm{~F}-\mathrm{FDG}-\mathrm{PET} / \mathrm{CT}$ in initial staging compared to conventional imaging (Eugene 2012; Federico 2012; Kumar 2010; Ricard 2011; Tateishi 2007). Unfortunately, FDG uptake is not unique to cancer cells. In addition, an 18F-FDG-PET scan visualises physiological FDG uptake in tissues such as the brain, brown adipose tissue, and the thymus, and tissues with inflammation and infection, causing increased glucose metabolism (Quak 2011).

\section{Clinical pathway}

Depending on the localisation of the tumour, patients present with a range of clinical symptoms. Patients with head and neck tumours can present with asymptomatic masses, proptosis, epistaxis, cranial nerve palsies, or chronic otitis media, whereas those with a tumour located in the bladder/prostate region could present with haematuria, urinary retention, abdominal mass, and constipation.

The diagnosis of RMS is confirmed by histology obtained by biopsy. The standard workup of newly diagnosed RMS patients includes a magnetic resonance image (MRI) of the primary tumour and several conventional imaging modalities to determine the extent of the disease, as follows.

- To exclude bone and bone marrow involvement, investigation involves a whole-body $99 \mathrm{mT}$ c bone scintigraphy and bilateral bone marrow aspirates and trephine biopsies.

- Lung metastases are identified with chest CT scan.

- To identify suspected lymph nodes, MRI of the primary tumour site is performed, and if indicated, an ultrasound of the regional lymph nodes is made.

- In patients with a parameningeal tumour, a lumbar puncture is indicated.

As the majority of newly diagnosed RMS patients are under six years of age (Yang 2014), general anaesthesia is indicated to obtain the results of different staging tests, including MRI and bone marrow punctures and trephines.

It is common practice that findings are discussed at a multidisciplinary tumour board meeting.

Based on histology (ARMS/ERMS), tumour site and size, postsurgical stage, nodal status, presence of distant metastasis, and age, patients are assigned to a risk group, and treatment decisions are made accordingly.

Patients diagnosed with metastatic disease will receive a more intense chemotherapeutic regimen compared to those with local disease. Evidence of regional lymph node involvement defined as those appropriate to the primary tumour site are not classified as patients with metastatic tumours. However, when nodal involvement beyond the regional lymph nodes is identified, the patient should be treated according to a protocol for metastatic disease, which also has consequences for local therapy approach. An example of regional lymph node involvement is the involvement

Fluorine-18-fluorodeoxyglucose (FDG) positron emission tomography (PET) computed tomography (CT) for the detection of bone, lung, $\quad 5$ and lymph node metastases in rhabdomyosarcoma (Review)

Copyright (c) 2021 The Cochrane Collaboration. Published by John Wiley \& Sons, Ltd. 
of inguinal nodes in a patient with a tumour located in the leg. In such a case, iliac or periaortic lymph nodes are classified as distant metastases.

\section{Alternative test(s)}

One disadvantage of $18 \mathrm{~F}$-FDG-PET/CT, especially in children, is the radiation exposure when multiple follow-up scans are indicated. Radiation exposure caused by repetitive CTs has been associated with brain tumours (Meulepas 2019), showing an excessive risk ratio per $100 \mathrm{mGy}$ of 0.86 . Radiation exposure is dependent on several factors, such as age of the child and the area of interest, and with newer-generation scanners and new reconstruction techniques the radiation dose has been reduced (Deak 2010; Padole 2015). This radiation burden can be further reduced when PET/MRI is used instead (Partovi 2014). The value of PET/MRI for diagnosis, staging follow-up, and therapy assessment for different paediatric malignancies needs to be further evaluated and is not within the scope of this review. The use of whole-body MRI including diffusionweighted imaging has shown to be another potential alternative for 18F-FDG-PET/CT in paediatric lymphoma (Littooij 2014).

\section{Rationale}

One of the aims of the current treatment protocols is to identify patients with a good prognosis so that they are not overtreated, and to make sure that patients with a poorer prognosis receive a more aggressive treatment regimen to obtain the best overall survival with the lowest late effects of treatment. The main risk stratification systems used at the moment to allocate treatment include site, size of the primary tumour, IRS postsurgical stage, age at diagnosis, nodal status, and presence of distant metastases (Bisogno 2018; Crist 2001; NCT00354835; NCT00379457; Oberlin 2008; Sultan 2010).

One of the disadvantages of the current workup at diagnosis is that many different imaging modalities are being used, and anaesthesia is often needed. Another disadvantage of the currently employed imaging modalities is that metastases could be located outside the field of view of the imaging technique used.

$18 \mathrm{~F}-\mathrm{FDG}-\mathrm{PET} / \mathrm{CT}$ is increasingly used in the diagnostic and staging process of sarcoma, including RMS. ${ }^{18}$ F-FDG-PET/CT may have sufficient sensitivity and specificity to identify bone and bone marrow metastases, lung metastases, and lymph node involvement. This might lead to adequate stratification of patients with RMS, and subsequently to application of adequate treatment intensity, duration, and modalities, with the advantage of using the 18 F-FDG-PET/CT as a single diagnostic test for the detection of metastases.

The objective of this Cochrane Review was to systematically assess all diagnostic accuracy data on the use of 18F-FDG-PET/CT in the diagnostic and staging process of patients with RMS at first diagnosis to detect metastases in order to assess the efficacy of this method in the diagnostic workup.

\section{O B JECT IVES}

To determine the diagnostic accuracy of 18F-FDG-PET/CT imaging for the detection of bone, lung, and lymph node metastases in RMS patients at first diagnosis.

\section{METHODS}

\section{Criteria for considering studies for this review}

\section{Types of studies}

Prospective or retrospective cross-sectional studies that report the diagnostic accuracy of $18 \mathrm{~F}-\mathrm{FDG}$-PET/CT in diagnosing lymph node involvement or bone or lung metastases or a combination of these metastases in patients with confirmed RMS were eligible for inclusion. Studies needed to compare the results of the 18F-FDG$\mathrm{PET} / \mathrm{CT}$ imaging with the tests described as reference standards (see Reference standards). The general criteria used for considering studies for this review are shown in Figure 1. Studies needed to report sufficient data to construct (part of) a $2 \times 2$ table, so the absolute number of true positives, false positives, true negatives, false negatives, or a combination of these had to be available from the data in the primary studies or obtained from study authors to reassess sensitivity and specificity. We excluded review articles, editorials or letters, and case reports. 
Figure 1. Criteria used to define studies eligible for this review. 18F-FDG-PET/CT: fluorine-18-fluorodeoxyglucose - positron emission tomography/computed tomography; CT: computed tomography; MRI: magnetic resonance imaging; RMS: rhabdomyosarcoma.

A Bone metastases

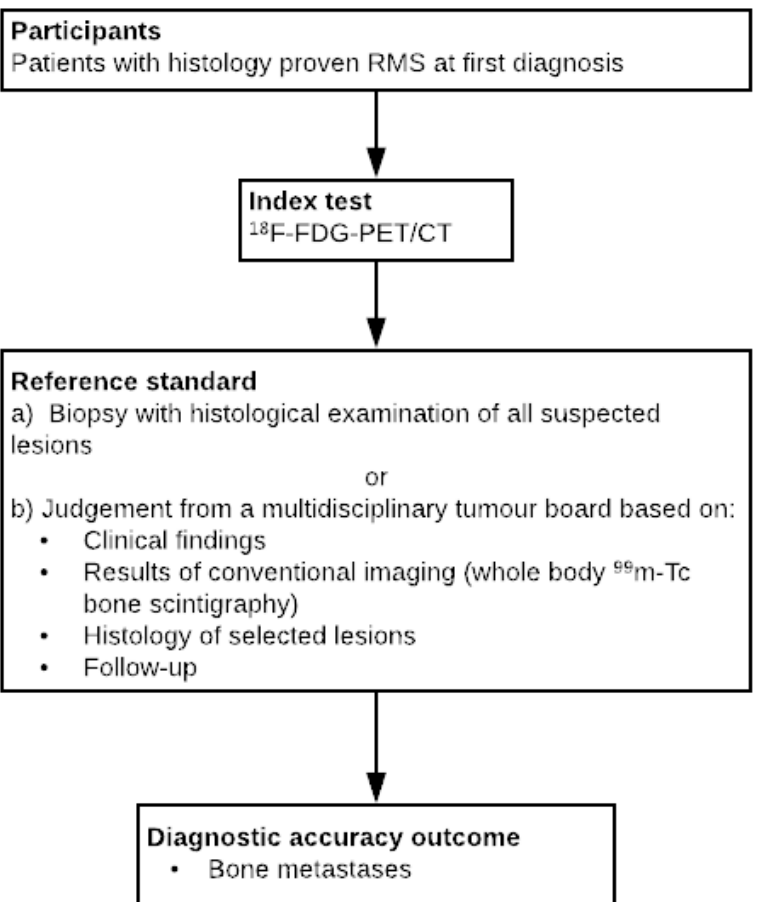

C Nodal involvement

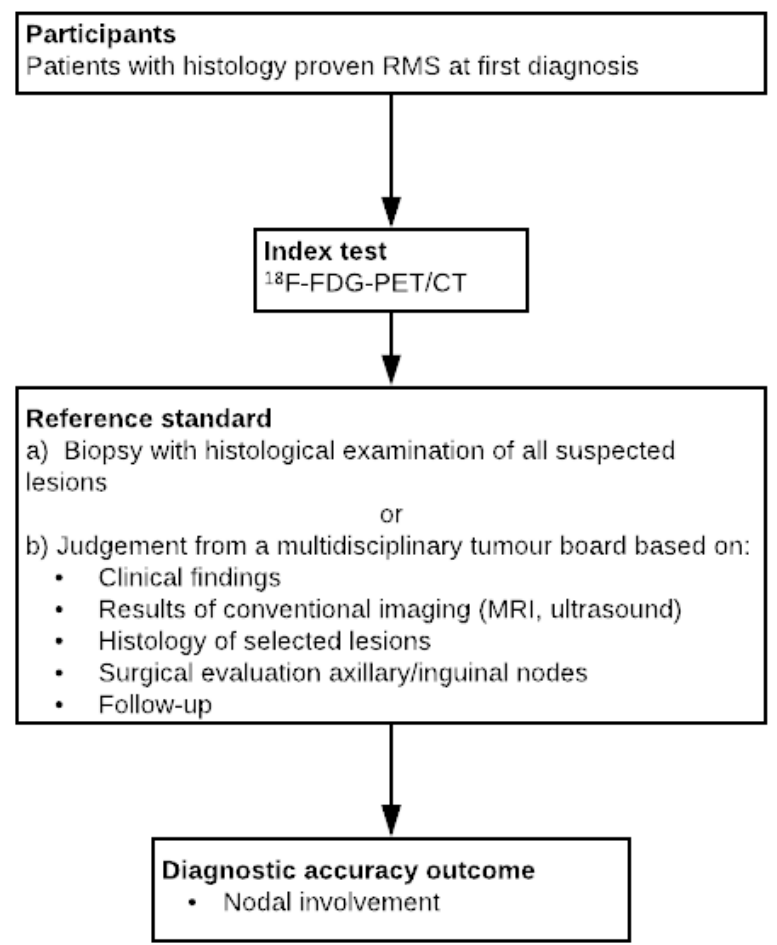

B Lung metastases

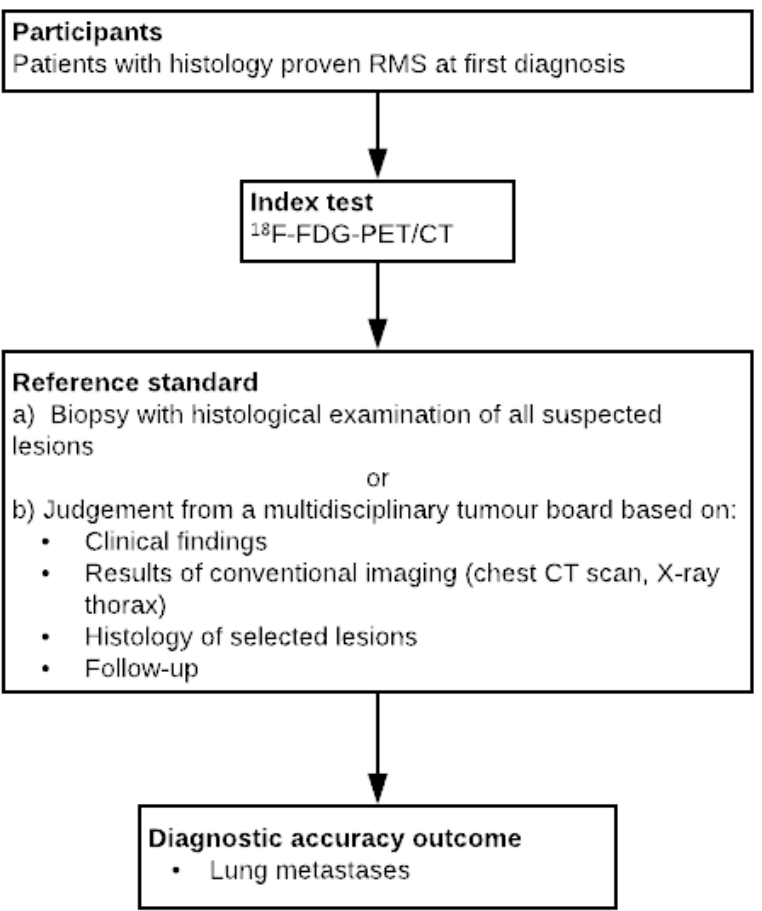

Fluorine-18-fluorodeoxyglucose (FDG) positron emission tomography (PET) computed tomography (CT) for the detection of bone, lung, and lymph node metastases in rhabdomyosarcoma (Review)

Copyright (c) 2021 The Cochrane Collaboration. Published by John Wiley \& Sons, Ltd. 


\section{Participants}

Participants with histologically confirmed RMS of any stage at first diagnosis. We included studies with participants who were not eligible for this review (such as those with recurrence of RMS or other sarcoma types) if data for only the eligible participants were available.

\section{Index tests}

\section{F-FDG-PET/CT scans.}

\section{Target conditions}

Newly diagnosed RMS with:

- bone metastases;

- lung metastases;

- nodal involvement;

- any combination of the above.

\section{Reference standards}

The optimal reference standard for suspected distant metastases and lymph node involvement in RMS patients would be confirmation by histopathology obtained by biopsy. For both ethical and practical reasons, this cannot be done for every suspected lesion.

When biopsy results were not available, the results of the $18 \mathrm{~F}$ FDG-PET/CT should have been compared with the judgement of a multidisciplinary tumour board, where experts had the knowledge of a patient's clinical findings, results from conventional imaging, and histological data. Clinical follow-up and imaging followup could also be used to support the final diagnosis of nodal involvement and bone and lung metastases (see Figure 1). In general, after nine weeks of chemotherapy, tumour response was evaluated with imaging including an X-ray of the thorax.

\section{Bone and bone marrow involvement}

A whole-body $99 \mathrm{~m}-\mathrm{Tc}$ skeleton scintigraphy and bilateral bone marrow aspirates and trephine biopsies were performed to identify bone metastases and bone marrow involvement. When possible, in case of doubt a biopsy is performed.

\section{Lung metastases}

Lung metastases are detected by chest CT scan of diagnostic quality. In most patients, an X-ray of the thorax was also performed.

Pulmonary metastatic disease was defined as one or more pulmonary nodules of 10-millimetre or more diameter or two or more well-defined nodules of 5- to 10-millimetre diameter, in the absence of another medical explanation. In case of doubt or 5 or more small (less than $5 \mathrm{~mm}$ ) nodules, a multidisciplinary tumour board decides whether a biopsy was indicated to confirm the diagnosis.

\section{Nodal involvement}

The presence of loco-regional nodal involvement was evaluated using MRI and ultrasound. In case of doubt, a biopsy was performed. In addition to such conventional imaging modalities, for upper and lower limb tumours, it is highly recommended to have surgical evaluation of axillary (for upper limb tumours) or inguinal (for lower limb tumours) nodes, even if nodes were clinically or radiological normal.

\section{Search methods for identification of studies}

Cochrane Childhood Cancer ran the searches in MEDLINE and Embase; all other searches were run by the review authors. We did not impose any language restrictions. We will update the searches every two years.

\section{Electronic searches}

We searched two electronic databases: MEDLINE in PubMed (from 1966 to 23 December 2020) and Embase in Ovid (from 1980 to 23 December 2020).

The search strategies for the different electronic databases (using a combination of controlled vocabulary and text words) are shown in Appendix 1 and Appendix 2.

\section{Searching other resources}

We located information about studies not indexed in MEDLINE and Embase, either published or unpublished, by handsearching the reference lists of relevant articles and review articles. We also contacted the authors of the included studies and other experts in the field of RMS for information about any ongoing or unpublished studies. We scanned conference proceedings electronically if available and otherwise by handsearching; we searched the International Society for Paediatric Oncology (SIOP), the American Society of Pediatric Hematology/Oncology (ASPHO), the Connective Tissue Oncology Society (CTOS), the American Society of Clinical Oncology (ASCO), and the European MusculoSkeletal Oncology Society (EMSOS) from 2014 to 2020. In Embase, we used the search fields conference publication (cg) and conference information (cf) in combination with Emtree terms and text words as mentioned in Appendix 2.

\section{Data collection and analysis}

\section{Selection of studies}

After employing the search strategy described above, two review authors independently identified studies meeting the inclusion criteria. We obtained in full text any study that seemed to meet the inclusion criteria based on title, abstract, or both. Two review authors independently undertook full-text article screening. We performed study selection using the data management platform Covidence (Covidence).

Only full-text studies that fulfilled all of the predefined criteria for considering studies for this review were eligible for inclusion. We clearly stated reasons for exclusion of any studies excluded at full-text review stage. Any disagreements during both initial selection and definite selection were resolved by consensus or by using a third-party arbitrator if necessary. We included a flow chart illustrating the selection of studies in the review (Figure 2). 
Figure 2. Study flow diagram. Abbreviations: ASCO, the American Society of Clinical Oncology; ASPHO, the American Society of Pediatric Hematology/Oncology; CTOS, the Connective Tissue Oncology Society; EMSOS, the European Musculo-Skeletal Oncology Society; SIOP, the International Society for Paediatric Oncology.

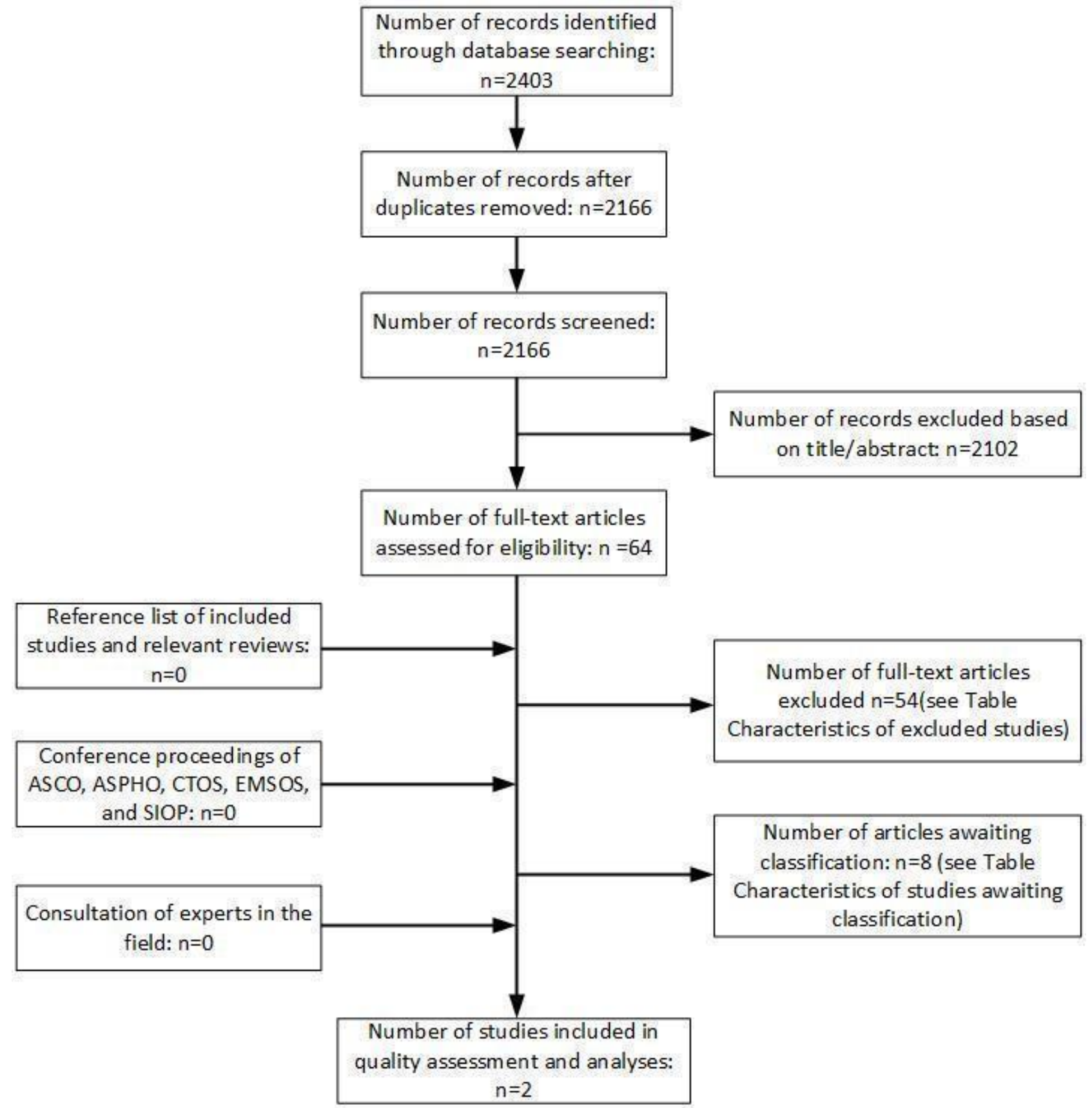

\section{Data extraction and management}

Two review authors independently performed data extraction using a predefined data extraction form. We extracted data on the following items.

- Article: author, year of publication (or presentation), journal (or conference).

- Study population: age at diagnosis, sex, histology (ARMS/ERMS), fusion status (PAX3/7-FOXO1), primary tumour site, IRS group (I, II, III), nodal status, metastasis status (bone, lung, other), number of participants (including number eligible for the study, number enrolled in the study, number receiving the index test and reference standard, number for whom results are reported in the $2 \times 2$ table, reasons for withdrawal).

- Index test: 18 F-FDG-PET/CT scan including the system and protocol used and the definition of an $18 \mathrm{~F}-\mathrm{FDG}-\mathrm{PET} / \mathrm{CT}$ positive lesion. Interpretation blinded to reference standards.

- Conventional imaging modalities used (MRI, CT, or both of the primary site, chest $\mathrm{CT}$ scan, chest $\mathrm{X}$-ray, radionucleotide bone

Fluorine-18-fluorodeoxyglucose (FDG) positron emission tomography (PET) computed tomography (CT) for the detection of bone, lung,

Copyright (c) 2021 The Cochrane Collaboration. Published by John Wiley \& Sons, Ltd. 
scan, craniospinal MRI, ultrasound abdomen, CT and/or MRI abdomen).

- Reference standard: description of the reference standard used. Verification of findings by: biopsy of suspected lesions or judgement of an interdisciplinary tumour board (based on combination of clinical findings, results from conventional imaging, additional biopsy and follow-up).

- Study design: basic design of the study (prospective cohort or historical cohort with data collection based on medical records or case-control study), time span between index test and reference test, treatment between index test and reference test.

- Data for the $2 \times 2$ table: true-positive, false-positive, truenegative, and false-negative rates or, if not available, relevant parameters (sensitivity, specificity or predictive values) to reconstruct the $2 \times 2$ table.

We piloted the data extraction form using two studies. There was a high concordance between the review authors, therefore we concluded that the form could be used for all studies.

When data were missing in a published report, we attempted to contact the authors for the missing information. In case of disagreement, we re-examined the abstracts and articles and undertook discussion until we achieved consensus. If this was not possible, we achieved final resolution using a third-party arbitrator.

\section{Assessment of methodological quality}

Two review authors independently assessed each included study for methodological quality. To do so, we adapted a four-domain tool from Quality Assessment of Diagnostic Accuracy Studies 2 (QUADAS-2) for our review (Whiting 2011). The tool comprises the following domains.

- Participant selection

- Index test

- Reference standard

- Flow of participants through the study and timing of both the index text and reference standard (flow and timing)

For each domain, we classified the risk of bias and concerns about the applicability of study findings as low, high, or unclear. See Table 1.

For example, for the participant selection domain, we evaluated whether a consecutive or random enrolment of participants had taken place. Some studies may have performed $18 \mathrm{~F}-\mathrm{FDG}-\mathrm{PET} / \mathrm{CT}$ solely in participants with unclear results obtained with standard tests, which could be a potential source of bias.

Any discrepancies between review authors were resolved by consensus or by using a third-party arbitrator if this was not possible.

We presented the methodological quality in the text, a graph, and in tables.

\section{Statistical analysis and data synthesis}

We performed a participant-based analysis of the data, that is sensitivity and specificity were calculated per participant instead of per lesion. We analysed data for the three separate outcomes (lung and bone metastases and nodal involvement) separately.
We used the data from the $2 \times 2$ tables (consisting of true positives, false positives, true negatives, and false negatives) to calculate sensitivity and specificity for each study and each test. We generated a paired forest plot showing estimates of sensitivity and specificity together with $95 \%$ confidence intervals. Such a forest plot provides a visual impression of the precision by which sensitivity and specificity have been measured in each study as well as an indication of the amount of variability in these parameters across studies.

\section{Investigations of heterogeneity}

We had planned to investigate heterogeneity using metaregression, by incorporating anticipated sources of heterogeneity in the bivariate model, but the limited number of studies prevented this. However, when assessing study results, we considered methodological and clinical sources of heterogeneity as well as variation in the criteria used to define a positive test result. Anticipated sources of heterogeneity included 18F-FDG-PET/CT protocol (e.g. FDG dose), participant population (e.g. percentage of alveolar histology), and reference standard (biopsy confirmed or not).

\section{Sensitivity analyses}

We had planned to perform sensitivity analyses, but the paucity of data prevented this.

\section{Assessment of reporting bias}

We undertook no formal assessment of reporting bias. However, we highlighted the possibility of reporting bias and interpreted the results of any analysis cautiously.

\section{RES U LTS}

\section{Results of the search}

We performed the electronic search on 23 December 2020. The electronic database searches identified a total of 2403 records. After removal of duplicates, we screened 2166 records based on title and abstract (see Figure 2). We excluded 2102 references for the following reasons: studies were review articles, editorials or letters, or case reports; studies were on animals; or studies were not performed in participants with newly diagnosed RMS. We evaluated 64 studies in full text, two of which fulfilled our inclusion criteria. We excluded 54 studies after full-text assessment; the reasons for exclusion are provided in Characteristics of excluded studies. For eight studies we needed additional information to determine whether or not they were eligible for inclusion; we assessed these studies as awaiting classification (see Characteristics of studies awaiting classification). We further examined the reference lists of the included studies, review articles, and conference proceedings of the above-mentioned organisations; this revealed no additional studies. A further consultation of experts in the field did not reveal any ongoing studies regarding this subject.

\section{Included studies}

The characteristics of the included studies are summarised in Characteristics of included studies and Table 2.

Both included studies were single-centre retrospective cohort studies. One study, performed in France (Eugene 2012), included participants with histologically proven RMS, with a median 
age at diagnosis of 8.7 years. A total of 23 participants were included. Participants underwent a chest radiograph, contrastenhanced $\mathrm{CT}$, and contrast-enhanced MRI of the primary tumour site and additional regions when clinically indicated, $99 \mathrm{mTc}$ bone scintigraphy, bone marrow biopsy, and an 18F-FDG-PET/CT as staging investigations. All images retrieved by conventional imaging modalities were reviewed by two reviewers blinded for results of $18 \mathrm{~F}-\mathrm{FDG}-\mathrm{PET} / \mathrm{CT}$. All $18 \mathrm{~F}-\mathrm{FDG}-\mathrm{PET} / \mathrm{CT}$ images were reviewed by two experienced readers blinded for results of conventional imaging modalities.

The other study (Ricard 2011), also performed in France, included participants with histologically proven RMS, with a median age at diagnosis of 9.6 years. A total of 13 participants were included. The included participants underwent an MRI of the primary tumour; contrast-enhanced CT of the thorax, abdomen, and pelvis area; 99mTc bone scintigraphy; bone marrow biopsy; and an 18F-FDG$\mathrm{PET} / \mathrm{CT}$ at diagnosis, except for one participant for whom only a chest CT and abdominal ultrasound and an 18F-FDG-PET/CT were performed. Whole-body $18 \mathrm{~F}-\mathrm{FDG}-\mathrm{PET} / \mathrm{CT}$ including limbs was only performed in case of a primary tumour located in the limbs. All images retrieved by conventional imaging modalities were reviewed by two nuclear physicians and a radiologist blinded for results of $18 \mathrm{~F}$-FDG-PET/CT. All 18F-FDG-PET/CT images were analysed by two nuclear medicine physicians blinded for results of conventional imaging modalities.

In both studies (Eugene 2012; Ricard 2011), histology was used as reference standard if available; in the case that histologic confirmation was not obtained (number of participants with histologic confirmation was not specified), the results of the multidisciplinary tumour board served as the reference standard.

All included participants underwent an 18F-FDG-PET/CT at initial diagnosis. The interval between conventional imaging and $18 \mathrm{~F}$ -
FDG-PET/CT was less than 15 days in the study of Ricard 2011. The mean time interval between conventional imaging and $18 \mathrm{~F}-\mathrm{FDG}$ $\mathrm{PET} / \mathrm{CT}$ was four days in the study of Eugene 2012. The administered dose of $18 \mathrm{~F}-\mathrm{FDG}$ varied from 3 to $7 \mathrm{MBq} / \mathrm{kg}$, and images were acquired 60 to 80 minutes after intravenous injection of 18 F-FDG. Ricard 2011 reported that the $18 \mathrm{~F}-\mathrm{FDG}-\mathrm{PET} / \mathrm{CT}$ was from head to upper thigh; a whole-body CT (head to toes) was only performed if the primary tumour was located in the extremities. Eugene 2012 reported that whole-body 18 F-FDG-PET/CT was performed; however, the field of view was not further specified. Ricard 2011 did not present a definition of a positive 18F-FDG-PET/CT lesion. Eugene 2012 defined a positive 18 F-FDG-PET/CT lesion as abnormal 18 F-FDG uptake greater than that of surrounding (adjacent) tissue without a known physiologic explanation. The interpretation of 18F-FDG-PET/CT imaging was done by two experienced observers in both studies (Eugene 2012; Ricard 2011).

\section{Excluded studies}

We excluded 54 studies (see Characteristics of excluded studies) for the following reasons: 26 studies used the wrong study design; 8 studies were not diagnostic studies; 7 studies were review articles; 6 studies either included no participants with RMS or only one participant with RMS; 5 studies were conference proceedings of which the full study was also evaluated for inclusion; 1 study was a duplicate publication (in French, primary publication in English excluded because wrong study design was used); and 1 study included participants that were also included in another publication.

\section{Methodological quality of included studies}

The quality assessments of the included studies are provided in Characteristics of included studies. An overview of the quality assessment according to the adapted QUADAS- 2 tool is shown in Figure 3 and Figure 4. 
Figure 3. Risk of bias and applicability concerns summary: review authors' judgements about each domain for each included study.

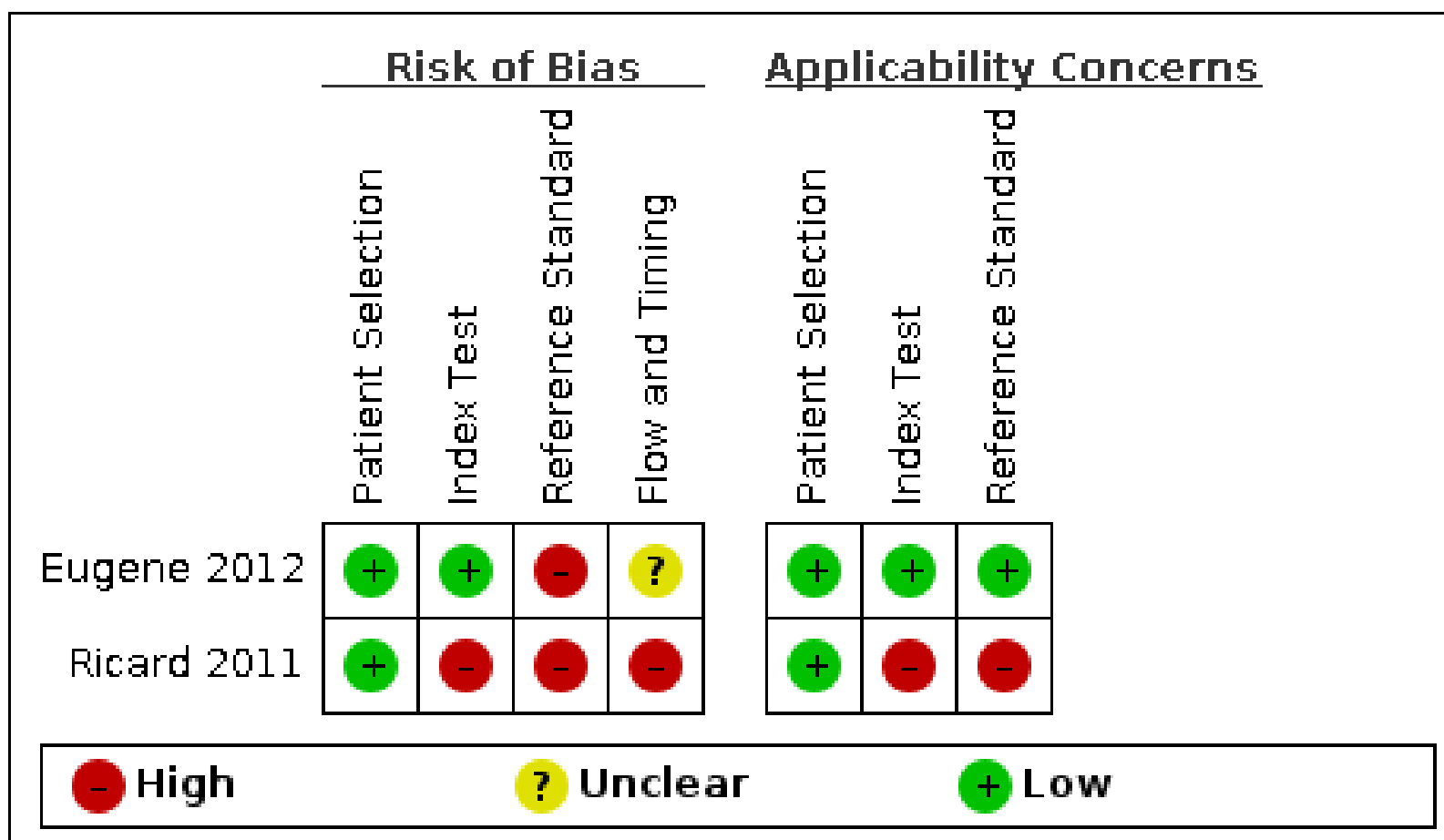

Figure 4. Risk of bias and applicability concerns graph: review authors' judgements about each domain presented as percentages across included studies.

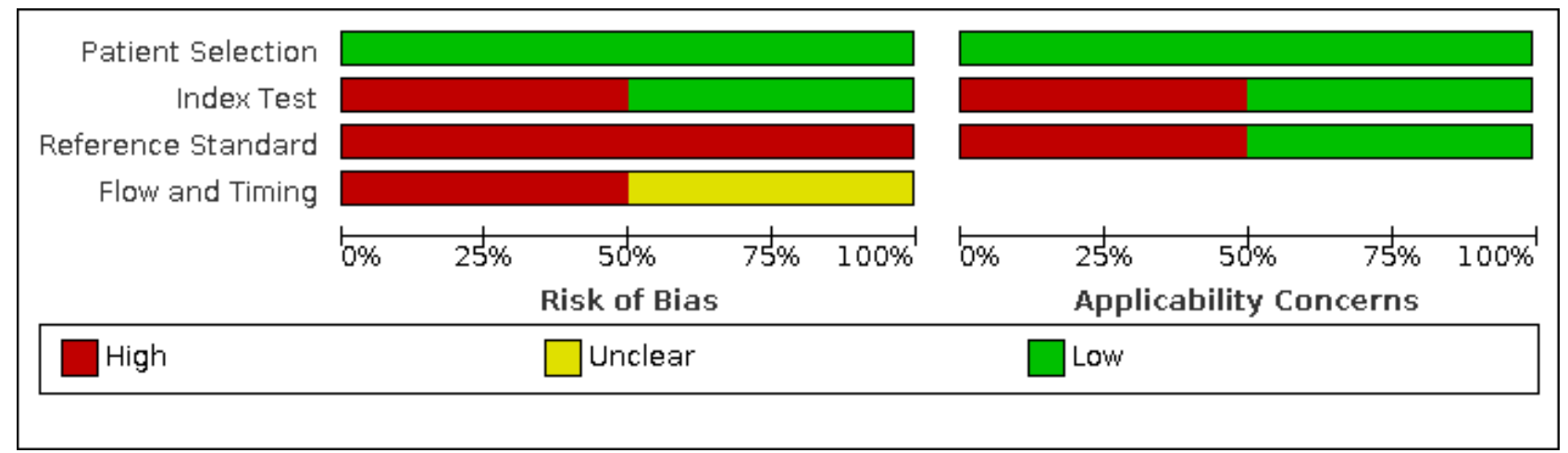

In summary, the selection of participants in both studies introduced a low risk of selection bias, and we judged the included participants and settings to be applicable to the review question.

Eugene 2012 reported a clear definition and cut-off of a positive lesion on FDG PET/CT, whereas this was not reported in Ricard 2011. This might have introduced bias and resulted in applicability concerns and problems regarding reproducibility.

Reference standard in both studies was comparable; however, both studies used multidisciplinary tumour boards as the reference standard when biopsy results were not available, in which results of $18 \mathrm{~F}$-FDG-PET/CT imaging were also taken into account. We therefore considered the risk of bias high for the reference standard domain. In Ricard 2011, 1 of 13 participants did not undergo all staging imaging tests, which also raised applicability concerns. We considered risk of bias regarding flow and timing high for Ricard 2011, whereas in Eugene 2012 the time between index test and reference test was not reported, resulting in a judgement of unclear for this domain.

\section{Findings}

Given the scarcity of data and heterogeneity between the included studies, a formal meta-analysis of diagnostic accuracy was not considered relevant. We were able to estimate the sensitivity and specificity of $18 \mathrm{~F}-\mathrm{FDG}-\mathrm{PET} / \mathrm{CT}$ using data from all the participants in the included studies ( $\mathrm{n}=36$ in total) and for all our predefined accuracy outcomes, except sensitivity for lung metastases, which

Fluorine-18-fluorodeoxyglucose (FDG) positron emission tomography (PET) computed tomography (CT) for the detection of bone, lung, 12 and lymph node metastases in rhabdomyosarcoma (Review)

Copyright $\odot 2021$ The Cochrane Collaboration. Published by John Wiley \& Sons, Ltd. 
was not estimable in Eugene 2012 since no participants had lung metastases; see Figure 5.

Figure 5. Forest plot for the accuracy of $18 \mathrm{~F}-\mathrm{FDG}-\mathrm{PET} / \mathrm{CT}$ for the detection of bone metastases, lung metastases, and nodal involvement. Sensitivity for lung metastases based on one true-positive and one false-positive lesion, therefore not reported in text.

${ }^{18}$ F-FDG-PET/CT for detection of bone metastases

$\begin{array}{lrrrrr}\text { Study } & \text { TP } & \text { FP } & \text { FN } & \text { TN } & \text { Sensitivity }(95 \% \mathrm{Cl}) \\ \text { Eugene } 2012 & 3 & 0 & 0 & 20 & 1.00[0.29,1.00] \\ \text { Ricard 2011 } & 4 & 0 & 0 & 9 & 1.00[0.40,1.00]\end{array}$

${ }^{18}$ F-FDG PET/CT for detection of lung metastases
Specificity $(95 \% \mathrm{Cl})$ Sensitivity $(95 \%$ Cl)Specificity $(95 \% \mathrm{Cl})$

$1.00[0.83,1.00]$

$1.00[0.66,1.00]$

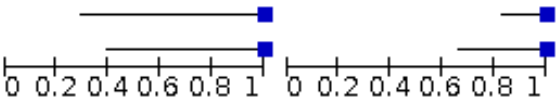

Specificity (95\% Cl) Sensitivity (95\% Cl)Specificity $(95 \% \mathrm{Cl})$ $0.96[0.78,1.00]$

$1.00[0.72,1.00]$

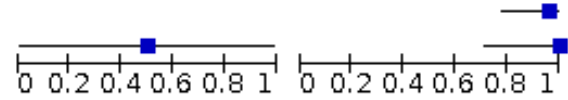

${ }^{18}$ F-FDG PET/CT for detection of nodal involvement

$\begin{array}{lrrrrr}\text { Study } & \text { TP } & \text { FP } & \text { FN } & \text { TN } & \text { Sensitivity }(95 \% \mathrm{Cl}) \\ \text { Eugene 2012 } & 8 & 0 & 0 & 15 & 1.00[0.63,1.00] \\ \text { Ricard 2011 } & 4 & 1 & 0 & 8 & 1.00[0.40,1.00]\end{array}$

Specificity (95\% Cl) Sensitivity (95\% Cl)Specificity $(95 \% \mathrm{Cl})$

$1.00[0.78,1.00]$

$0.89[0.52,1.00]$

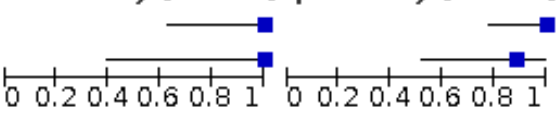

Due to the limited number of included studies we were not able to investigate heterogeneity. However, we observed potential sources of heterogeneity such as the number of participants with alveolar histology (39\% in Eugene 2012 and 66\% in Ricard 2011) and potential differences in the definition of a positive $18 \mathrm{~F}-\mathrm{FDG}-\mathrm{PET} /$ CT lesion (Eugene 2012 used abnormal uptake greater than that of surrounding background not explained by normal organ uptake, whilst in Ricard 2011 this was not specified).

\section{Bone metastases}

Both studies reported the diagnostic accuracy of $18 \mathrm{~F}-\mathrm{FDG}-\mathrm{PET} / \mathrm{CT}$ for the detection of bone metastases (Eugene 2012; Ricard 2011). A total of 7 out of 36 participants (19\%) were considered to have bone metastases at presentation. The reported sensitivity and specificity was $100 \%$ ( $95 \%$ confidence interval (Cl) for sensitivity was $29 \%$ to $100 \%$ in Eugene 2012 and $40 \%$ to $100 \%$ in Ricard 2011; $95 \% \mathrm{Cl}$ for specificity was $83 \%$ to $100 \%$ for Eugene 2012 and $66 \%$ to $100 \%$ for Ricard 2011) in both studies.

\section{Lung metastases}

Both studies reported the diagnostic accuracy of 18 F-FDG-PET/CT for the detection of lung metastases (Eugene 2012; Ricard 2011). A total of 2 out of 36 participants (5.6\%) were considered to have lung metastases at presentation. Participants included in Eugene 2012 did not have lung metastases, therefore sensitivity could not be estimated. Two participants had lung metastases in Ricard 2011, one of which was detected by 18 F-FDG-PET/CT imaging. Reported specificity was $96 \%(95 \% \mathrm{Cl} 78 \%$ to $100 \%)$ in Eugene 2012 and $100 \%(95 \% \mathrm{Cl} 72 \%$ to $100 \%)$ in Ricard 2011 . In one participant in Eugene 2012, 18F-FDG-PET/CT imaging showed a positive lung lesions, which turned out to be of infectious aetiology.

\section{Nodal involvement}

Both studies reported the diagnostic accuracy of 18 F-FDG-PET/CT for the detection of nodal involvement (Eugene 2012; Ricard 2011). A total of 12 out of 36 participants (33\%) were diagnosed with nodal involvement at presentation. The reported sensitivity in both studies was $100 \%$ (95\% Cl 63\% to 100\% in Eugene 2012 and 40\% to $100 \%$ in Ricard 2011). The reported specificity was $100 \%$ (95\% $\mathrm{Cl} 78 \%$ to $100 \%$ ) in Eugene 2012 and $89 \%$ (95\% Cl 52\% to 100\%) in Ricard 2011. In one participant in Ricard 2011, 18F-FDG-PET/CT imaging showed a false-positive lymph node, potentially caused by a concurrent infection.

\section{DISCUSSION}

\section{Summary of main results}

In this Cochrane Diagnostic Test Accuracy Review we assessed the diagnostic accuracy of 18 F-FDG-PET/CT for the detection of bone metastases and lung metastases and lymph node involvement in RMS at first diagnosis. Only two small studies fulfilled all our inclusion criteria, which impeded formal meta-analysis of accuracy outcomes. Across these two studies we have reported the sensitivity and specificity of $18 \mathrm{~F}-\mathrm{FDG}-\mathrm{PET} / \mathrm{CT}$, as follows.

- The sensitivity (determined in 7 participants) and specificity (determined in 29 participants) of 18F-FDG-PET/CT for the detection of bone metastases was $100 \%$ (95\% Cl for sensitivity: $29 \%$ to $100 \%$ for Eugene 2012 and $40 \%$ to $100 \%$ for Ricard 2011 ; 95\% Cl for specificity: $83 \%$ to $100 \%$ for Eugene 2012 and $66 \%$ to $100 \%$ for Ricard 2011) (Figure 5; Summary of findings 1).

- The sensitivity of $18 \mathrm{~F}-\mathrm{FDG}-\mathrm{PET} / \mathrm{CT}$ for the detection of lung metastases was not reported, since data were only available for two participants, one of which was identified with metastases. 
Specificity was 96\% for Eugene 2012 (95\% Cl 78\% to 100\%) and $100 \%$ for Ricard 2011 (95\% Cl 72\% to 100\%) (Figure 5; Summary of findings 1 ).

- The sensitivity (determined in 12 participants) of 18 F-FDG-PET/ CT for the detection of lymph node involvement was 100\% (95\% Cl $63 \%$ to $100 \%$ for Eugene 2012 and $40 \%$ to $100 \%$ for Ricard 2011). Specificity (determined in 24 participants) was $100 \%$ for Eugene 2012 (95\% Cl 78\% to 100\%) and 89\% for Ricard 2011 (95\% Cl 52\% to $100 \%$ ) (Figure 5; Summary of findings 1 ).

\section{Strengths and weaknesses of the review}

The results of this review provide a clear overview of the currently available evidence regarding the accuracy of 18 F-FDG$\mathrm{PET} / \mathrm{CT}$ for the detection of bone and lung metastases and lymph node involvement in newly diagnosed RMS. Two review authors independently identified studies and extracted the data, according to the protocol of this review (Breunis 2016). We did not impose any language restrictions and included abstracts; however, the impact of publication bias on these results could not be determined since determinants for publication bias in diagnostic test accuracy studies are not well known (van Enst 2014).

\section{Reference standard}

The optimal reference standard for suspected distant metastases and lymph node involvement in patients with RMS is histopathologic confirmation by biopsy. However, this cannot be done for every suspected lesion. We therefore also included studies in which, when biopsy results were not available, the results of $18 \mathrm{~F}-\mathrm{FDG}-\mathrm{PET} / \mathrm{CT}$ were compared with the judgement of multidisciplinary tumour boards together with clinical and imaging follow-up. We considered this as the reference standard for this review because it reflects clinical practice. However, in this circumstance, the results of $18 \mathrm{~F}$-FDG-PET/CT imaging are also available to the multidisciplinary tumour boards, therefore the reference standard might also have been influenced by the index test, potentially resulting in incorporation bias (Kohn 2013).

We excluded several studies reporting on the diagnostic accuracy of $18 \mathrm{~F}$-FDG-PET/CT in participants with RMS because they just compared results of $18 \mathrm{~F}-\mathrm{FDG}-\mathrm{PET} / \mathrm{CT}$ with conventional imaging, which was not within the scope of this review. This resulted in a very limited number of included studies.

\section{Scarcity of the available evidence}

The most important limitation of this review was the lack of available data. We identified only 2 studies, encompassing a total of 36 participants, which precluded meta-analysis. The results of this review should therefore be interpreted with great caution.

Both included studies were retrospective, single-centre studies, including a maximum of 23 participants per study. Due to these small numbers, and because the number of participants with metastatic disease was even lower, one 18F-FDG-PET/CT scan more or less scored as false negative would have had a large impact on sensitivity. The inclusion of a small number of participants might also explain the differences in participant characteristics between those included in Ricard 2011 (13 participants) and larger series on RMS, such as Weiss $2013(n=1687)$. The majority of participants in Ricard 2011 (77\%) had alveolar RMS, whereas this was 35\% in Weiss 2013.
However, participants were only included if they had received both 18F-FDG-PET/CT imaging and conventional imaging. It is unclear whether referral for ${ }^{18}$ F-FDG-PET/CT imaging was based on the a priori likelihood of metastases. This might explain why the included studies reported a high percentage of participants with bone metastases ( $7 / 36$ participants (19\%)), whereas this was $5 \%$ in Weiss 2013 , suggesting potential referral bias in the studies included in our review (Kohn 2013).

In this review we performed a participant-based analysis of the accuracy of $18 \mathrm{~F}-\mathrm{FDG}-\mathrm{PET} / \mathrm{CT}$ for the detection of lymph node involvement and bone and lung metastases, because one positive metastatic lesion is enough to classify patients as having metastatic disease. However, reported sensitivity using participantbased data is probably higher than expected for a lesion-based analysis. Moreover, accurate classification of all metastatic lesions is necessary to apply adequate local therapy regimens.

\section{Eligibility of studies awaiting classification}

We were not able to assess the eligibility of eight studies for inclusion in this review. We attempted to contact the study authors to obtain additional information, but were unsuccessful. The impact of this on the outcomes of our review is unclear; however, it remains uncertain whether these studies would have fulfilled our inclusion criteria.

\section{Applicability of findings to the review question}

The findings of this review show the paucity of evidence regarding the diagnostic accuracy of 18F-FDG-PET/CT for the detection of bone and lung metastases and lymph node involvement in newly diagnosed RMS. The findings of this review are applicable to patients with newly diagnosed RMS only. Based on the available evidence, we could not reliably determine the accuracy of 18 F-FDG$\mathrm{PET} / \mathrm{CT}$ in the detection of bone and lung metastases and lymph node involvement in RMS.

\section{AUTHORS' CONCLUSIONS}

\section{Implications for practice}

Based on the available evidence from two included studies, we have concluded that there is insufficient evidence to reliably determine the diagnostic accuracy of $18 \mathrm{~F}$-FDG-PET/CT in the detection of bone and lung metastases and lymph node involvement in newly diagnosed patients with RMS. For clinical practice this implies that $18 \mathrm{~F}$-FDG-PET/CT cannot replace all other staging investigations as a single diagnostic test for metastases at present. Other studies assessing the value of $18 \mathrm{~F}-\mathrm{FDG}-\mathrm{PET} / \mathrm{CT}$ in the staging of newly diagnosed patients with RMS show promising results. However, these studies generally lacked a gold standard such as histology or tumour board evaluation, making it impossible to determine the diagnostic accuracy, and were therefore excluded from the review.

Although we could not determine the diagnostic accuracy of $18 \mathrm{~F}$ FDG-PET/CT in RMS, 18 F-FDG-PET/CT is extensively used in staging investigations for newly diagnosed patients with RMS. In current treatment protocols, $18 \mathrm{~F}-\mathrm{FDG}-\mathrm{PET} / \mathrm{CT}$ has replaced $99 \mathrm{mTc}$ bone scintigraphy for the detection of bone metastases. The results on the accuracy of 18F-FDG-PET/CT to detect bone metastases are promising, since the studies included in this review reported a 
$100 \%$ sensitivity and $89 \%$ to $100 \%$ specificity of $18 \mathrm{~F}-\mathrm{FDG}-\mathrm{PET} / \mathrm{CT}$ to detect bone metastases. However, larger, prospective studies on the accuracy of ${ }^{18} \mathrm{~F}-\mathrm{FDG}-\mathrm{PET} / \mathrm{CT}$ are needed to confirm these findings.

In this review we evaluated the diagnostic accuracy of 18F-FDG$\mathrm{PET} / \mathrm{CT}$ for the detection of bone and lung metastases and lymph node involvement in RMS, whereas the accuracy of $18 \mathrm{~F}-\mathrm{FDG}$-PET/CT to detect metastases in other sites, such as soft-tissue metastases, was not assessed. 18F-FDG-PET/CT might play a pivotal role in identifying these lesions. For this reason, it is important that $18 \mathrm{~F}$ FDG-PET/CT be done from head to toe, instead of head to upper thigh, as was the case in Ricard 2011.

\section{Implications for research}

Larger series evaluating the diagnostic accuracy of 18F-FDG-PET/ $\mathrm{CT}$ for the detection of bone and lung metastases and lymph node involvement in patients with newly diagnosed RMS are necessary.

Such studies might prove challenging to undertake, or even unethical, because RMS mainly affects young children, and because 18F-FDG-PET/CT is already established in the initial workup of patients with RMS in state-of-the-art study protocols (e.g. NCT00379457). A prospective study comparing the diagnostic accuracy of 18 F-FDG-PET/CT to whole-body $99 \mathrm{mT}$ Tc bone scintigraphy is not expected, because this would lead to additional radiation exposure.

Besides the use of 18F-FDG-PET/CT for the detection of lymph node involvement and bone and lung metastases, we expect that future studies will also focus on PET/MRI techniques because of limited radiation doses (Partovi 2014). This technique is relatively new and needs to be evaluated in paediatric malignancies. Furthermore, future studies should evaluate the addition of diffusion-weighted imaging to whole-body MRI as a potential alternative to ${ }^{18} \mathrm{~F}-\mathrm{FDG}$ PET for the staging of paediatric RMS, as was previously shown in paediatric lymphoma (Littooij 2014).

\section{ACK N O WLEDGEMENTS}

We would like to acknowledge the Editorial Base of Cochrane Childhood Cancer for their advice and support. We thank Cochrane Netherlands for their support. We thank Ina Monsef (Information Specialist, Cochrane Haematology) for running the search. We also thank the Diagnostic Test Accuracy Editorial Team and Prof Dr MM van Noesel, paediatric oncologist, Princess Máxima Center for Pediatric Oncology, Utrecht, who kindly agreed to peer review this review. The Editorial Base of Cochrane Childhood Cancer has been funded by Stichting Kinderen Kankervrij (KiKa) and is located at the Princess Máxima Center for Pediatric Oncology, Utrecht, the Netherlands. 


\section{RE F E R E N C E S}

\section{References to studies included in this review}

Eugene 2012 \{published data only\}

Eugene T, Corradini N, Carlier T, Dupas B, Leux C, Bodet-Milin C. 18F-FDG-PET/CT in initial staging and assessment of early response to chemotherapy of pediatric rhabdomyosarcomas. Nuclear Medicine Communications 2012;33(10):1089-95. [DOI: 10.1097/MNM.0b013e328356741f]

Ricard 2011 \{published data only\}

Ricard F, Cimarelli S, Deshayes E, Mognetti T, Thiesse P, Giammarile F. Additional benefit of F-18 FDG PET/CT in the staging and follow-up of pediatric rhabdomyosarcoma. Clinical Nuclear Medicine 2011;36(8):672-7. [DOI: 10.1097/ RLU.0b013e318217ae2e]

\section{References to studies excluded from this review}

Andersen 2015 \{published data only\}

Andersen KF, Fuglo HM, Rasmussen SH, Petersen MM, Loft A. Semi-quantitative calculations of primary tumor metabolic activity using F-18 FDG PET/CT as a predictor of survival in 92 patients with high-grade bone or soft tissue sarcoma. Medicine (Baltimore) 2015;94(28):e1142.

\section{Annovazzi 2020 \{published data only\}}

Annovazzi A, Rea S, Zoccali C, Sciuto R, Baldi J, Anelli V, et al. Diagnostic and clinical impact of 18F-FDG PET/CT in staging and restaging soft-tissue sarcomas of the extremities and trunk: mono-Institutional retrospective study of a sarcoma referral center. Journal of Clinical Medicine 2020;9(8):1-12.

\section{Arush 2007 \{published data only\}}

Arush MW, Israel O, Postovsky S, Militianu D, Meller I, Zaidman I, et al. Positron emission tomography/computed tomography with 18 fluoro-deoxyglucose in the detection of local recurrence and distant metastases of pediatric sarcoma. Pediatric Blood \& Cancer 2007;49(7):901-5.

\section{Baek 2015 \{published data only\}}

Baek S, Yoon D, Kim J. Soft tissue sarcoma of the head and neck: $\mathrm{CT}, \mathrm{MRI}$, and FDG-PET/CT imaging findings. European Journal of Nuclear Medicine and Molecular Imaging 2015;42 Suppl 1:S1-924.

\section{Bar-Sever 2007 \{published data only\}}

Bar-Sever Z, Keidar Z, Ben-Barak A, Bar-Shalom R, Postovsky S, Guralnik $L$, et al. The incremental value of $18 \mathrm{~F}$-FDG PET/CT in paediatric malignancies. European Journal of Nuclear Medicine and Molecular Imaging 2007;34(5):630-7.

\section{Baum 2010 \{published data only\}}

Baum S, Fruehwald M, Rahbar K, Wessling J, Schober O, Weckesser M. PET/(CT) and outcome in children and young adults with rhabdomyosarcoma. Journal of Nuclear Medicine 2010;51(Suppl 2):511.
Becher 2015 \{published data only\}

Becher S, Oskouei S. PET imaging in sarcoma. Orthopedic Clinics of North America 2015;46(3):409-15, xi.

Bentancourt 2016 \{published data only\}

Bentancourt C, Banchero A, Rossi S, Alonso O, Gaudiano J, Engler $\mathrm{H}$. Role of $18 \mathrm{~F}$-FDG PET/CT for initial staging and restaging of sarcomas. European Journal of Nuclear Medicine and Molecular Imaging 2016;43(Suppl 1):1-734.

Brisse 2009 \{published data only\}

Brisse HJ. Staging of common paediatric tumours. Pediatric Radiology 2009;39 Suppl 3:482-90.

Ceyssens 2011 \{published data only\} Ceyssens S, Stroobants S. Sarcoma. Methods in Molecular Biology 2011;727:191-203.

Charest 2009 \{published data only\}

Charest M, Hickeson M, Lisbona R, Novales-Diaz JA, Derbekyan V, Turcotte RE. FDG PET/CT imaging in primary osseous and soft tissue sarcomas: a retrospective review of 212 cases. European Journal of Nuclear Medicine and Molecular Imaging 2009;36(12):1944-51.

Daldrup-Link 2001 \{published data only\}

Daldrup-Link HE, Franzius C, Link TM, Laukamp D, Sciuk J, Jurgens $\mathrm{H}$, et al. Whole-body MR imaging for detection of bone metastases in children and young adults: comparison with skeletal scintigraphy and FDG PET. American Journal of Roentgenology 2001;177(1):229-36.

Dong 2017 \{published data only\}

Dong Y, Zhang X, Wang S, Chen S, Ma C. 18F-FDG PET/CT is useful in initial staging, restaging for pediatric rhabdomyosarcoma. Quarterly Journal of Nuclear Medicine and Molecular Imaging 2017;61(4):438-46.

Elkholy 2017 \{published data only\}

Elkholy E, Abd El-Giad S, Fathy H. How can FDG PET CT add benefits in staging pediatric rhabdomyosarcoma? European Journal of Nuclear Medicine and Molecular Imaging 2017;44(Suppl 2):119-956.

\section{Elmanzalawy 2020 \{published data only\}}

Elmanzalawy A, Vali R, Chavhan GB, Gupta AA, Omarkhail Y, Amirabadi A, et al. The impact of (18)F-FDG PET on initial staging and therapy planning of pediatric soft-tissue sarcoma patients. Pediatric Radiology 2020;50(2):252-60.

Eugene 2010 \{published data only\}

Eugene T, Ansquer C, Oudoux A, Corradini N, Carlier T, Thomas $\mathrm{C}$, et al. FDG PET/CT in initial staging and early response to chemotherapy assessment of paediatric rhabdomyosarcomas. Medecine Nucleaire-Imagerie Fonctionnelle Et Metabolique 2010;34(12):655-63. 


\section{Federico 2012 \{published data only\}}

Federico SM, McCarville B, Spunt S, Shulkin B, Krasin M, Billups C. Comparison of PET-CT and conventional imaging in staging pediatric rhabdomyosarcoma. Pediatric Blood \& Cancer 2012;58(7):1018-2012.

\section{Federico 2013 \{published data only\}}

Federico SM, Spunt SL, Krasin MJ, Billup CA, Wu J, Shulkin B, et al. Comparison of PET-CT and conventional imaging in staging pediatric rhabdomyosarcoma. Pediatric Blood \& Cancer 2013;60(7):1128-34

\section{Fuglo 2012 \{published data only\}}

Fuglo HM, Jorgensen SM, Loft A, Hovgaard D, Petersen MM. The diagnostic and prognostic value of (1)(8)F-FDG PET/CT in the initial assessment of high-grade bone and soft tissue sarcoma. A retrospective study of 89 patients. European Journal of Nuclear Medicine and Molecular Imaging 2012;39(9):1416-24.

\section{Gambhir 2016 \{published data only\}}

Gambhir S, Prashanth A, Pradhan P, Dixit M, Sankar G, Singh A, et al. Impact of PET-CT in soft-tissue sarcomas. Journal of Nuclear Medicine 2016;57(Suppl 2):1573.

\section{Gupta 2015 \{published data only\}}

Gupta RK Jr, Tripathi M, Bakshi S, Damle N, Kumar K, Bhayana R, et al. Evaluation of the role of F-18 FDG PET/CT in childhood rhabdomyosarcoma - preliminary results. European Journal of Nuclear Medicine and Molecular Imaging 2015;42 Suppl 1:S1-924.

\section{Hagi 2018 \{published data only\}}

Hagi T, Nakamura T, Sugino Y, Matsubara T, Asanuma K, Sudo A. Is FDG-PET/CT useful for diagnosing pulmonary metastasis in patients with soft tissue sarcoma? Anticancer Research 2018;38(6):3635-9.

\section{lagaru 2006 \{published data only\}}

Iagaru A, Chawla S, Menendez L, Conti PS. 18F-FDG PET and $\mathrm{PET} / \mathrm{CT}$ for detection of pulmonary metastases from musculoskeletal sarcomas. Nuclear Medicine Communications 2006;27(10):795-802.

\section{lagaru 2006a \{published data only\}}

lagaru A, Quon A, McDougall IR, Gambhir SS. F-18 FDG PET/CT evaluation of osseous and soft tissue sarcomas. Clinical Nuclear Medicine 2006;31(12):754-60.

\section{Kleis 2009 \{published data only\}}

Kleis M, Daldrup-Link H, Matthay K, Goldsby R, Lu Y, Schuster T, et al. Diagnostic value of PET/CT for the staging and restaging of pediatric tumors. European Journal of Nuclear Medicine and Molecular Imaging 2009;36(1):23-36.

\section{Klem 2007 \{published data only\}}

Klem ML, Grewal RK, Wexler LH, Schoder H, Meyers PA, Wolden SL. PET for staging in rhabdomyosarcoma: an evaluation of PET as an adjunct to current staging tools. Journal of Pediatric Hematology/Oncology 2007;29(1):9-14.

\section{Kumar 2008 \{published data only\}}

Kumar J, Seith A, Kumar A, Sharma R, Bakhshi S, Kumar R, et al. Whole-body MR imaging with the use of parallel imaging for detection of skeletal metastases in pediatric patients with small-cell neoplasms: comparison with skeletal scintigraphy and FDG PET/CT. Pediatric Radiology 2008;38(9):953-62.

Locantore 2013 \{published data only\}

Locantore L, Tredici M, Volterrani D, Paglianiti I, Betti F, Coccoli L, et al. Impact of 18 F-FDG PET/CT for staging, restaging and assessment of response to treatment in bone and soft tissue sarcomas. European Journal of Nuclear Medicine and Molecular Imaging 2013;40 Suppl 2:S89-564.

\section{Ma 2015 \{published data only\}}

Ma C. FDG PET/CT is useful in initial staging, restaging for pediatric rhabdomyosarcoma. Journal of Nuclear Medicine 2015;56 Suppl 3:421.

\section{Macpherson 2018 \{published data only\}}

Macpherson RE, Pratap S, Tyrrell H, Khonsari M, Wilson S, Gibbons M, et al. Retrospective audit of 957 consecutive (18)FFDG PET-CT scans compared to CT and MRI in 493 patients with different histological subtypes of bone and soft tissue sarcoma. Clinical Sarcoma Research 2018;8(1):9.

\section{Massardo 2012 \{published data only\}}

Massardo T, Jofre MJ, Sierralta MP, Canessa J, Castro G, Berrocal I, et al. Positron emission tomography with fluorinedeoxyglucose in sarcomas and non-sarcoma non-epithelial tumors. Revista Medica de Chile 2012;140(9):1116-25.

McCarville 2005 \{published data only\}

McCarville MB, Christie R, Daw NC, Spunt SL, Kaste SC. PET/CT in the evaluation of childhood sarcomas. American Journal of Roentgenology 2005;184(4):1293-304.

McCarville 2011 \{published data only\}

McCarville B, Krasin M, Spunt S, Billups C, Wu J, Shulkin B. $\mathrm{PET} / \mathrm{CT}$ in pediatric rhabdomyosarcoma. Pediatric Radiology 2011;41(S1):250-310.

\section{Mody 2010 \{published data only\}}

Mody RJ, Bui C, Hutchinson RJ, Yanik GA, Castle VP, Frey KA, et al. FDG PET imaging of childhood sarcomas. Pediatric Blood \& Cancer 2010;54(2):222-7.

\section{Murphy 2008 \{published data only\}}

Murphy JJ, Tawfeeq M, Chang B, Nadel H. Early experience with PET/CT scan in the evaluation of pediatric abdominal neoplasms. Journal of Pediatric Surgery 2008;43(12):2186-92.

\section{Piperkova 2009 \{published data only\}}

Piperkova E, Mikhaeil M, Mousavi A, Libes R, Viejo-Rullan F, Lin $\mathrm{H}$, et al. Impact of PET and CT in PET/CT studies for staging and evaluating treatment response in bone and soft tissue sarcomas. Clinical Nuclear Medicine 2009;34(3):146-50. 
Reichert 2004 \{published data only\}

Reichert B, Bahre M, Mailander P. Positron emission tomography (PET) in soft-tissue sarcoma. Handchirurgie Mikrochirurgie Plastische Chirurgie 2004;36(5):296-300.

\section{Ricard 2010 \{published data only\}}

Ricard F, Cimarelli S, Deshayes E, Mognetti T, Thiesse P, Giammarile F. Utility of FDG PET/CT in childhood rhabdomyosarcoma. European Journal of Nuclear Medicine and Molecular Imaging 2010;37(Suppl 2):S443.

\section{Sa 2020 \{published data only\}}

Sa R, Liu D, Zhao H, Hou S, Lin Q, Guan F. Utility of [(18)F] fluoro-deoxyglucose positron emission tomography/computed tomography for staging and therapy response evaluation in pediatric rhabdomyosarcoma: a case series and literature review. Frontiers in Medicine (Lausanne) 2020;7:281.

\section{Sciuto 2014 \{published data only\}}

Sciuto R, D'Angelo G, Annovazzi A, Bergomi S, Mazzone C, Pasqualoni $R$, et al. 18F-FDG PET/CT role in the clinical management of muscolo-skeletal tumors: a retrospective analysis on 720 studies. European Journal of Nuclear Medicine and Molecular Imaging 2014;41 Suppl 2:151-705.

\section{Sheikhbahaei 2015 \{published data only\}}

Sheikhbahaei S, Marcus C, Hafezi-Nejad N, Taghipour M, Subramaniam RM. Value of FDG PET/CT in patient management and outcome of skeletal and soft tissue sarcomas. PET Clinics 2015;10(3):375-93.

\section{Shin 2008 \{published data only\}}

Shin DS, Shon OJ, Han DS, Choi JH, Chun KA, Cho IH. The clinical efficacy of (18)F-FDG-PET/CT in benign and malignant musculoskeletal tumors. Annals of Nuclear Medicine 2008;22(7):603-9.

\section{Singhal 2014 \{published data only\}}

Singhal N, Qureshi S, Chinnaswami G, Kembhavi S, Rangarajan V, Desai S, et al. Role of PET-CT in staging of pediatric round cell tumors. Can it eliminate the need for bone marrow biopsy? Pediatric Blood \& Cancer 2014;61 Suppl 2:S105-433.

\section{Sorschag 2011 \{published data only\}}

Sorschag M, Malle P, Kohlfurst S, Lobnig M, Lind P, Gallowitsch HJ. F-18 FDG PET/CT in patients with osseous and soft tissue sarcoma. NuklearMedizin 2011;50(2):A13-4.

\section{Tabacchi 2016 \{published data only\}}

Tabacchi E, Fanti S, Nanni C. The possible role of PET imaging toward individualized management of bone and soft tissue malignancies. PET Clinics 2016;11(3):285-96.

\section{Tateishi 2007 \{published data only\}}

Tateishi U, Yamaguchi U, Seki K, Terauchi T, Arai Y, Kim EE. Bone and soft-tissue sarcoma: preoperative staging with fluorine 18 fluorodeoxyglucose PET/CT and conventional imaging. Radiology 2007;245(3):839-47.

\section{Tateishi 2009 \{published data only\}}

Tateishi U, Hosono A, Makimoto A, Nakamoto Y, Kaneta T, Fukuda $\mathrm{H}$, et al. Comparative study of FDG PET/CT and conventional imaging in the staging of rhabdomyosarcoma. Annals of Nuclear Medicine 2009;23(2):155-61.

\section{Terwisscha 2015 \{published data only\}}

Terwisscha Van Scheltinga S, Wijnen M, Heij H, Wijnen R, Van Baren R, Merks $\mathrm{H}$, et al. FDG-PET/CT improves staging in extremity rhabdomyosarcoma. Pediatric Blood \& Cancer 2015;62(S4):S143-418.

\section{Tezol 2020 \{published data only\}}

Tezol O, Sagcan F, Ozcan PP, Citak EC. Bone marrow involvement in pediatric malignancies: a comparison study of positron emission tomography-computed tomography and bone marrow biopsy. Turkish Journal of Pediatrics 2020;62(2):182-90

\section{Turpin 2016 \{published data only\}}

Turpin B, Pressey J, Nagarajan R, Gelfand M, Dasgupta R. The impact of sentinel lymph node evaluation in paediatric, adolescent and young adult head and neck rhabdomyosarcoma. Pediatric Blood \& Cancer 2016;63 Suppl 3:S5-321.

\section{Volker 2007 \{published data only\}}

Volker T, Denecke T, Steffen I, Misch D, Schonberger S, Plotkin M, et al. Positron emission tomography for staging of pediatric sarcoma patients: results of a prospective multicenter trial. Journal of Clinical Oncology 2007;25(34):5435-41.

\section{Wagner 2017 \{published data only\}}

Wagner LM, Kremer N, Gelfand MJ, Sharp SE, Turpin BK, Nagarajan R, et al. Detection of lymph node metastases in pediatric and adolescent/young adult sarcoma: sentinel lymph node biopsy versus fludeoxyglucose positron emission tomography imaging - a prospective trial. Cancer 2017;123(1):155-60.

\section{Zapata 2015 \{published data only\}}

Zapata C, Olavarrieta R, Raskin S, Cuglievan B, Desai K, DeAngulo G. The role of PET/CT vs bone marrow biopsy in the initial evaluation of bone marrow infiltration in various pediatric solid tumors. Pediatric Blood \& Cancer 2015;62(Suppl 2):106.

\section{Zapata 2018 \{published data only\}}

Zapata CP, Cuglievan B, Zapata CM, Olavarrieta R, Raskin S, Desai $\mathrm{K}$, et al. $\mathrm{PET} / \mathrm{CT}$ versus bone marrow biopsy in the initial evaluation of bone marrow infiltration in various pediatric malignancies. Pediatric Blood \& Cancer 2018;65(2):e26814.

\section{References to studies awaiting assessment}

\section{De Ferrater 2013 \{published data only\}}

De Ferrater MB, London K, Robert HG. FDG PET CT in paediatric head and neck cancer. Internal Medicine Journal 2013;43(Suppl 1):10. [DOI: 10.1111/imj.12132] 


\section{Mazurek 2011 \{published data only\}}

Mazurek A. Value of PET-CT in the evaluation of sarcomas in children. European Journal of Nuclear Medicine and Molecular Imaging 2011;38(Suppl 2):S387. [DOI: 10.1007/ s00259-011-1911-0]

\section{Nguyen 2011 \{published data only\}}

Nguyen JQ, Davis K, Mittra ES, Quon A, Gambhir SS, Marina N, et al. Clinical utility of 18F FDG PET/CT and 99mTc MDP bone scintigraphy in patients with Ewings sarcoma and other sarcomas. Clinical Nuclear Medicine 2011;36(7):620. [DOI: 10.1097/RLU.0b013e31821f0df0]

Oguz 2013 \{published data only\}

Oguz A, Okur A, Akdemir O, Karadeniz C, Pinarli FG, Tekkesin F, et al. Role of $18 \mathrm{~F}$-FDG PET CT in staging and remission evaluation of patients with pediatric solid tumors. Pediatric Blood \& Cancer 2013;60(Suppl 3):96. [DOI: 10.1002/pbc.24719]

Riad 2010 \{published data only\}

Riad R, Omar W, Aboskera T, Hussein SH, Mousa E, Ataia I, et al. The role and impact of F-18 FDG PET/CT on management of paediatric patients with head and neck cancer. European Journal of Nuclear Medicine and Molecular Imaging 2010;37(Suppl 2):S304. [DOI: 10.1007/s00259-010-1557-3]

\section{Sourabh 2010 \{published data only\}}

Moon Sourabh D, Mahajan S, Thapa P, Gupta P, Sahana Mishra AK, Jyotika J, et al. Role of F-18 FDG PET/CT in evaluation of bone and soft tissue sarcomas. Indian Journal of Nuclear Medicine 2010;25(3):92.

\section{Tuncel 2015 \{published data only\}}

Tuncel M, Kurucu N, Kiratli PO, Erbas B, Akyuz C. Clinical impact of FDG PET-CT in pediatric soft tissue sarcomas. European Journal of Nuclear Medicine and Molecular Imaging 2015;1(Suppl 1):S206. [DOI: 10.1007/s00259-015-3198-z]

\section{Walter 2012 \{published data only\}}

Walter F, Czernin J, Hall T, Allen-Auerbach M, Walter MA, Dunkelmann $\mathrm{S}$, et al. Is there a need for dedicated bone imaging in addition to $18 \mathrm{~F}-\mathrm{FDG} \mathrm{PET} / \mathrm{CT}$ imaging in pediatric sarcoma patients? Journal of Pediatric Hematology/Oncology 2012;34(2):131-6. [DOI: 10.1097/MPH.0b013e3182282825]

\section{Additional references}

\section{Arndt 2009}

Arndt CA, Stoner JA, Hawkins DS, Rodeberg DA, HayesJordan AA, Paidas CN, et al. Vincristine, actinomycin, and cyclophosphamide compared with vincristine, actinomycin, and cyclophosphamide alternating with vincristine, topotecan, and cyclophosphamide for intermediate-risk rhabdomyosarcoma: Children's Oncology Group Study D9803. Journal of Clinical Oncology 2009;27:5182-8.

\section{Arndt 2013}

Arndt CA. Risk stratification of rhabdomyosarcoma: a moving target. American Society of Clinical Oncology Educational Book 2013;33:415-9. [DOI: 10.1200/EdBook_AM.2013.33.415]

\section{Bisogno 2018}

Bisogno G, Jenney M, Bergeron C, Gallego Melcón S, Ferrari A, Oberlin O, et al. Addition of dose-intensified doxorubicin to standard chemotherapy for rhabdomyosarcoma (EpSSG RMS 2005): a multicentre, open-label, randomised controlled, phase 3 trial. Lancet Oncology 2018;19(8):1061-71.

\section{Covidence [Computer program]}

Veritas Health Innovation Covidence. Version accessed 4 February 2019. Melbourne, Australia: Veritas Health Innovation. Available at covidence.org.

\section{Crist 2001}

Crist WM, Anderson JR, Meza JL, Fryer C, Raney RB, Ruymann FB, et al. Intergroup rhabdomyosarcoma studyIV: results for patients with nonmetastatic disease. Journal of Clinical Oncology 2001;19(12):3091-102. [DOI: 10.1200/ JCO.2001.19.12.3091]

\section{Deak 2010}

Deak PD, Smal Y, Kalender WA. Multisection CT protocols: sexand age-specific conversion factors used to determine effective dose from dose-length product. Radiology 2010;257(1):158-66.

\section{Gallamini 2014}

Gallamini A, Zwarthoed C, Borra A. Positron emission tomography (PET) in oncology. Cancers (Basel) 2014;6(4):1821-89. [DOI: 10.3390/cancers6041821]

\section{Gambhir 2002}

Gambhir SS. Molecular imaging of cancer with positron emission tomography. Nature Reviews Cancer 2002;2(9):683-93. [DOI: $10.1038 / \mathrm{nrc882}$ ]

\section{Gatta 2014}

Gatta G, Botta L, Rossi S, Aareleid T, Bielska-Lasota M, Clavel J, et al. Childhood cancer survival in Europe 1999-2007: results of EUROCARE-5 - a population-based study. Lancet Oncology 2014;15(1):35-47. [DOI: 10.1016/S1470-2045(13)70548-5]

\section{Kohn 2013}

Kohn MA, Carpenter CR, Newman TB. Understanding the direction of bias in studies of diagnostic test accuracy. Academic Emergency Medicine 2013;20(11):1194-206.

\section{Kumar 2010}

Kumar R, Shandal V, Shamim SA, Halanaik D, Malhotra A. Clinical applications of PET and PET/CT in pediatric malignancies. Expert Review of Anticancer Therapy 2010;10:755-68.

\section{Littooij 2014}

Littooij AS, Kwee TC, Barber I, Granata C, Vermoolen MA, Enríquez G, et al. Whole-body MRI for initial staging of paediatric lymphoma: prospective comparison to an FDGPET/CT-based reference standard. European Radiology 2014;24(5):1153-65.

\section{McDowell 2003}

McDowell HP. Update on childhood rhabdomyosarcoma. Archives of Disease in Childhood 2003;88:354-7. 


\section{Meulepas 2019}

Meulepas JM, Ronckers CM, Smets AMJB, Nievelstein RAJ, Gradowska P, Lee C, et al. Radiation exposure from pediatric CT scans and subsequent cancer risk in the Netherlands. Journal of the National Cancer Institute 2019;111(3):256-63.

\section{Meza 2006}

Meza JL, Anderson J, Pappo AS, Meyer WH. Analysis of prognostic factors in patients with nonmetastatic rhabdomyosarcoma treated on intergroup rhabdomyosarcoma studies III and IV: the Children's Oncology Group. Journal of Clinical Oncology 2006;24:3844-51.

\section{Miller 1995}

Miller RW, Young JL, Novakovic B. Childhood cancer. Cancer 1995;75(1 Suppl):395-405.

\section{NCT00354835}

NCT00354835. Randomized study of vincristine, dactinomycin and cyclophosphamide (VAC) versus VAC alternating with vincristine and irinotecan (VI) for patients with intermediaterisk rhabdomyosarcoma (RMS). clinicaltrials.gov/show/ NCT00354835 (first received 20 July 2006).

\section{NCT00379457}

NCT00379457. A protocol for nonmetastatic rhabdomyosarcoma [RMS-2005]. clinicaltrials.gov/show/ NCT00379457 (first received 21 September 2006).

\section{Oberlin 2008}

Oberlin O, Rey A, Lyden E, Bisogno G, Stevens MC, Meyer WH, et al. Prognostic factors in metastatic rhabdomyosarcomas: results of a pooled analysis from United States and European cooperative groups. Journal of Clinical Oncology 2008;26:2384-9.

\section{Padole 2015}

Padole A, Ali Khawaja RD, Kalra MK, Singh S. CT radiation dose and iterative reconstruction techniques. American Journal of Roentgenology 2015;204(4):W384-92.

\section{Pappo 2007}

Pappo AS, Lyden E, Breitfeld P, Donaldson SS, Wiener E, Parham D, et al. Two consecutive phase II window trials of irinotecan alone or in combination with vincristine for the treatment of metastatic rhabdomyosarcoma: the Children's Oncology Group. Journal of Clinical Oncology 2007;25:362-9.

\section{Partovi 2014}

Partovi S, Kohan A, Rubbert C, Vercher-Conejero JL, Gaeta C, Yuh R, et al. Clinical oncologic applications of PET/MRI: a new horizon. American Journal of Nuclear Medicine and Molecular Imaging 2014;4:202-12.

\section{Quak 2011}

Quak E, van de Luijtgaarden AC, de Geus-Oei LF, van der Graaf WT, Oyen WJ. Clinical applications of positron emission tomography in sarcoma management. Expert Review of Anticancer Therapy 2011;11:195-204.

\section{Raney 2001}

Raney RB, Maurer HM, Anderson JR, Andrassy RJ, Donaldson SS, Qualman SJ, et al. The Intergroup Rhabdomyosarcoma Study Group (IRSG): major lessons from the IRS-I through IRS-IV studies as background for the current IRS-V treatment protocols. Sarcoma 2001;5:9-15.

\section{Raney 2011}

Raney RB, Walterhouse DO, Meza JL, Andrassy RJ, Breneman JC, Crist WM, et al. Results of the Intergroup Rhabdomyosarcoma Study Group D9602 protocol, using vincristine and dactinomycin with or without cyclophosphamide and radiation therapy, for newly diagnosed patients with low-risk embryonal rhabdomyosarcoma: a report from the Soft Tissue Sarcoma Committee of the Children's Oncology Group. Journal of Clinical Oncology 2011;29:1313-8.

\section{Sultan 2010}

Sultan I, Ferrari A. Selecting multimodal therapy for rhabdomyosarcoma. Expert Review of Anticancer Therapy 2010;10:1285-301.

\section{van Enst 2014}

van Enst WA, Ochodo E, Scholten RJ, Hooft L, Leeflang MM. Investigation of publication bias in meta-analyses of diagnostic test accuracy: a meta-epidemiological study. BMC Medical Research Methodology 2014;14(1):70.

\section{Ward 2014}

Ward E, DeSantis C, Robbins A, Kohler B, Jemal A. Childhood and adolescent cancer statistics, 2014. CA: a Cancer Journal for Clinicians 2014;64:83-103.

\section{Weiss 2013}

Weiss AR, Lyden ER, Anderson JR, Hawkins DS, Spunt SL, Walterhouse DO, et al. Histologic and clinical characteristics can guide staging evaluations for children and adolescents with rhabdomyosarcoma: a report from the Children's Oncology Group Soft Tissue Sarcoma Committee. Journal of Clinical Oncology 2013;31:3226-32.

\section{Whiting 2011}

Whiting PF, Rutjes AW, Westwood ME, Mallett S, Deeks J, Reitsma JB, et al. QUADAS-2: a revised tool for the quality assessment of diagnostic accuracy studies. Annals of Internal Medicine 2011;18:529-38.

\section{Yang 2014}

Yang L, Takimoto T, Fujimoto J. Prognostic model for predicting overall survival in children and adolescents with rhabdomyosarcoma. BMC Cancer 2014;14:654.

\section{References to other published versions of this review Breunis 2016}

Breunis WB, Haveman LM, Vaarwerk B, Owers EC, van Rijn RR, van den Berg $\mathrm{H}$, et al. Fluorine-18-fluorodeoxyglucose (FDG) positron emission tomography (PET) computed tomography (CT) for the detection of bone, lung and lymph node metastases in rhabdomyosarcoma. Cochrane Database 
of Systematic Reviews 2016, Issue 8. Art. No: CD012325. [DOI:

10.1002/14651858.CD012325]

CHARACTERISTICS OF STUDIES

Characteristics of included studies [ordered by study ID]

Eugene 2012

\section{Study characteristics}

Patient Sampling

Inclusion period: 2003 to 2010

Patient population: all children treated for histologically proven RMS at University Hospital of Nantes (France)

Consecutive or random sample: consecutive patients who underwent a whole-body 18F-FDG-PET/CT before therapy initiation

Patient characteristics and setting

Retrospective cohort study

Number of patients eligible: not reported

Number of patients enrolled: $n=23$

Number of patients for whom results are reported: $n=23$

Diagnostic workup: conventional imaging (chest radiograph, contrast-enhanced CT, and contrast-enhanced MRI of primary tumour site and additional regions when clinically indicated, $99 \mathrm{mTc}$ bone scintigraphy), and bone marrow biopsy

Median age at diagnosis: 8.7 years, range: 9 months to 21.6 years

Sex distribution: 16 males (70\%), 7 females (30\%)

Histology: ARMS: $n=9$ (39\%), ERMS: $n=13$ (57\%), botryoid RMS: $n=1(4 \%)$

Fusion status: fusion positive: $\mathrm{n}=6$ (26\%), fusion negative: $\mathrm{n}=3(13 \%)$

Nodal status: locoregional, $n=4(17 \%)$; distant lymph nodes, $n=4(17 \%)$

Metastasis status: bone metastases, $n=3(13 \%)$, lung metastases, $n=0(0 \%)$, other not reported

Primary tumour site: orbit, $n=5(22 \%)$; parameningeal, $n=5(22 \%)$; head/neck nonparameningeal, $n=2$ (9\%); genito-urinary bladder prostate, $n=4(17 \%)$; limbs, $n=5$ (22\%); other, $\mathrm{n}=1$ (4\%); unknown, $\mathrm{n}=1(4 \%)$

Index tests

$$
\begin{aligned}
& \text { Whole-body } 18 \text { F-FDG-PET/CT images were acquired using a Discovery LS PET/CT imag- } \\
& \text { ing system (GE Medical Systems) or mCT Biograph imaging system (Siemens). } \\
& \text { Intravenous injection of } 5 \text { to } 7 \mathrm{MBq} / \mathrm{kg} \text { of } 18 \mathrm{~F}-\mathrm{FDG} \text { or intravenous injection } 3 \mathrm{MBq} / \mathrm{kg} \\
& 18 \mathrm{~F}-\mathrm{FDG} 60 \text { to } 80 \text { minutes before imaging } \\
& \text { Children fasted for at least } 4 \text { hours before } 18 \mathrm{~F}-\mathrm{FDG} \text { injection, and blood glucose level } \\
& \text { controlled before injection. } \\
& \text { Images evaluated in consensus by } 2 \text { experienced readers. } \\
& \text { Positive test result: abnormal uptake greater than that of surrounding background not } \\
& \text { explained by normal organ uptake }
\end{aligned}
$$

\footnotetext{
Target condition and reference standard(s) Target condition: newly diagnosed, histologically proven RMS
} 
Conventional imaging: chest radiograph, contrast-enhanced CT, and contrast-enhanced MRI of primary tumour site and additional regions when clinically indicated, 99mTc bone scintigraphy

Reference standard: the results of conventional imaging modalities, bone marrow biopsy, and 18F-FDG-PET/CT were finally verified by an interdisciplinary tumour board.

All staging examinations, histopathology of biopsies and resected specimens, and clinical data including the serial follow-up examinations were used.

Flow and timing

All patients received the same reference standard.

Average time between index test and reference standard was 4 days.

No treatment between index test and reference standard

\section{Comparative}

\section{Notes}

Additional information provided by study authors.

\section{Methodological quality}

\begin{tabular}{llll}
\hline Item Authors' judgement & Risk of bias & Applicability concerns \\
\hline
\end{tabular}

DOMAIN 1: Patient Selection Was a consecutive or random sample of Yes
patients enrolled?

\begin{tabular}{ll}
\hline Was a case-control design avoided? & Yes \\
\hline $\begin{array}{l}\text { Did the study avoid inappropriate exclu- } \\
\text { sions? }\end{array}$ & Yes
\end{tabular}

Could the selection of patients have in- Low risk
troduced bias?

Are there concerns that the included pa-
tients and setting do not match the re-
view question?

\section{DOMAIN 2: Index Test (All tests)}

Were the index test results interpreted Yes without knowledge of the results of the reference standard?

If a threshold was used, was it pre-speci- Yes fied?

Did the study provide a clear definition of Yes what was considered to be a positive test result?

Were uninterpretable/intermediate test re- Unclear sults reported?

\section{Could the conduct or interpretation of the index test have introduced bias?}


Eugene 2012 (Continued)

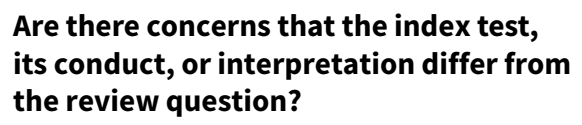

\section{DOMAIN 3: Reference Standard}

Is the reference standards likely to correct- Yes

ly classify the target condition?

Were the reference standard results interpreted without knowledge of the results of the index tests?

\section{Could the reference standard, its con- duct, or its interpretation have intro- duced bias?}

\section{Are there concerns that the target con- dition as defined by the reference stan- dard does not match the question?}

\section{DOMAIN 4: Flow and Timing}

Did all patients receive the same reference Yes standard?

\begin{tabular}{l} 
Were all patients included in the analysis? Yes \\
$\begin{array}{l}\text { Was the delay between the performance Unclear } \\
\text { of the 18F-FDG-PET/CT and the reference } \\
\text { standard less than } 2 \text { weeks? }\end{array}$ \\
$\begin{array}{l}\text { Could the patient flow have introduced } \\
\text { bias? }\end{array}$ \\
\hline
\end{tabular}

\section{Ricard 2011}

\section{Study characteristics}

Patient population: all patients aged 1 to 20 years at diagnosis of histologically proven RMS admitted to hospital in Lyon (France)

Consecutive or random sample: consecutive patients who underwent staging with conventional imaging and 18F-FDG-PET/CT before systemic therapy

Number of patients eligible: not reported

Number of patients enrolled: $\mathrm{n}=13$

Number of patients for whom results are reported: $n=13$

Diagnostic workup: MRI of the primary tumour, contrast-enhanced CT of the thorax, abdomen and pelvis area, $99 \mathrm{mTc}$ bone scintigraphy 
Ricard 2011 (Continued)

Median age at diagnosis: 9.6 years, range: 1.8 to 19.1 years

Sex distribution: 12 males (92\%), 1 female (8\%)

Histology: ARMS: $n=10$ (77\%), ERMS: $n=3(23 \%)$

Fusion status: not reported

Nodal status: locoregional, $n=4(31 \%)$; distant lymph nodes, $n=1(8 \%)$

Metastasis status: bone metastases, $n=4(31 \%)$, lung metastases, $n=2(15 \%)$, other not reported

Primary tumour site: parameningeal, $n=2(15 \%)$; head/neck non-parameningeal, $n=4(31 \%)$; genito-urinary, $n=3(23 \%)$; limbs, $n=4(31 \%)$

Index tests

18F-FDG-PET/CT images were acquired on a Philips Gemini PET/CT system after intravenous injection of $5 \mathrm{MBq} / \mathrm{kg}$ of FDG. Images were acquired approximately 60 minutes after tracer injection.

Head-to-upper-thigh CT scan, only whole body if RMS located in the extremity

Analysed by 2 nuclear medicine physicians blinded to results of $\mathrm{CI}$. SUVmax was measured in positive primary lesions.

Positive test result: not specified

Target condition and reference standard(s)

Target condition: newly diagnosed, histologically proven RMS

Conventional imaging: MRI of the primary tumour, contrast-enhanced CT of the thorax, abdomen and pelvis area, $99 \mathrm{mTc}$ bone scintigraphy

Reference standard: when $\mathrm{Cl}$ and $18 \mathrm{~F}$-FDG-PET/CT produced discordant results, the patient's histologic data and final clinical evaluation of the multidisciplinary tumour board were considered as the reference standard

Flow and timing

1 patient only underwent chest $\mathrm{CT}$ and abdominal ultrasound as conventional imaging.

Time between index test and reference standard: $<15$ days

No treatment between index test and reference standard

Comparative

Notes

We could not contact the study authors.

\section{Methodological quality}

\begin{tabular}{llll}
\hline Item & Authors' judgement & Risk of bias & Applicability concerns \\
\hline
\end{tabular}

\section{DOMAIN 1: Patient Selection}

Was a consecutive or random sample of patients Yes enrolled?

Was a case-control design avoided?

Yes

Did the study avoid inappropriate exclusions?

Yes

Fluorine-18-fluorodeoxyglucose (FDG) positron emission tomography (PET) computed tomography (CT) for the detection of bone, lung, 
Ricard 2011 (Continued)

Could the selection of patients have introduced bias?
Low risk

Low concern

Are there concerns that the included patients and setting do not match the review question?

\section{DOMAIN 2: Index Test (All tests)}

Were the index test results interpreted without

Yes

knowledge of the results of the reference standard?

If a threshold was used, was it pre-specified? Unclear

Did the study provide a clear definition of what was No

considered to be a positive test result?

Were uninterpretable/intermediate test results re- Unclear ported?

Could the conduct or interpretation of the index
test have introduced bias?

test have introduced bias?

Are there concerns that the index test, its con-

duct, or interpretation differ from the review

question?

\section{DOMAIN 3: Reference Standard}

Is the reference standards likely to correctly classi- No

fy the target condition?

Were the reference standard results interpreted No

without knowledge of the results of the index tests?

$\begin{array}{ll}\text { Could the reference standard, its conduct, or its } & \text { High risk } \\ \text { interpretation have introduced bias? } & \end{array}$

interpretation have introduced bias?

\section{Are there concerns that the target condition as defined by the reference standard does not match the question?}

\section{DOMAIN 4: Flow and Timing}

Did all patients receive the same reference standard?

Were all patients included in the analysis?

Yes

Was the delay between the performance of the $18 \mathrm{~F}-\quad$ Yes FDG-PET/CT and the reference standard less than 2 weeks?

\section{Could the patient flow have introduced bias?}

High risk

Abbreviations: 18F-FDG-PET/CT: fluorine-18-fluorodeoxyglucose - positron emission tomography/computed tomography; $99 \mathrm{mTC}$ bone scintigraphy: technetium-99m bone scintigraphy; ARMS: alveolar rhabdomyosarcoma; $\mathrm{Cl}$ : conventional imaging; CT: computed 
tomography; ERMS: embryonal rhabdomyosarcoma; MRI: magnetic resonance imaging; RMS: rhabdomyosarcoma; SUVmax: standardised uptake value.

Characteristics of excluded studies [ordered by study ID]

\begin{tabular}{|c|c|}
\hline Study & Reason for exclusion \\
\hline Andersen 2015 & No patients with rhabdomyosarcoma \\
\hline Annovazzi 2020 & $\begin{array}{l}\text { Wrong study design: compared FDG-PET/CT results to histology or follow-up imaging, instead of } \\
\text { multidisciplinary tumour board }\end{array}$ \\
\hline Arush 2007 & Wrong study design: $\mathrm{PET} / \mathrm{CT}$ performed at time of relapse \\
\hline Baek 2015 & Study was not primary diagnostic. \\
\hline Bar-Sever 2007 & Wrong study design: compared FDG-PET/CT to FDG-PET \\
\hline Baum 2010 & Study was not primary diagnostic. \\
\hline Becher 2015 & No original research: review \\
\hline Bentancourt 2016 & Wrong study design: no comparison described \\
\hline Brisse 2009 & No original research: review \\
\hline Ceyssens 2011 & No original research: review \\
\hline Charest 2009 & Wrong study design: only focused on diagnostic accuracy of primary tumour \\
\hline Daldrup-Link 2001 & Wrong study design: no FDG-PET/CT performed \\
\hline Dong 2017 & Wrong study design: compared FDG-PET to conventional imaging \\
\hline Elkholy 2017 & Wrong study design: compared FDG-PET to conventional imaging \\
\hline Elmanzalawy 2020 & Wrong study design: compared FDG-PET to conventional imaging \\
\hline Eugene 2010 & Duplicate publication of Eugene 2012; primary study included \\
\hline Federico 2012 & Conference proceeding; full report evaluated \\
\hline Federico 2013 & Wrong study design: 1 study investigator compared results between $\mathrm{Cl}$ and FDG-PET \\
\hline Fuglo 2012 & $\begin{array}{l}\text { Wrong study design: compared FDG-PET to conventional imaging. No separate results for patients } \\
\text { with rhabdomyosarcoma }\end{array}$ \\
\hline Gambhir 2016 & Study was not primary diagnostic. \\
\hline Gupta 2015 & Wrong study design: compared FDG-PET/CT to FDG/PET \\
\hline Hagi 2018 & No patients with rhabdomyosarcoma \\
\hline lagaru 2006 & Wrong study design: compared FDG-PET to CT \\
\hline lagaru 2006a & $\begin{array}{l}\text { Wrong study design: compared FDG-PET to conventional imaging. No separate results for patients } \\
\text { with rhabdomyosarcoma }\end{array}$ \\
\hline
\end{tabular}

Fluorine-18-fluorodeoxyglucose (FDG) positron emission tomography (PET) computed tomography (CT) for the detection of bone, lung, 


\begin{tabular}{|c|c|}
\hline Study & Reason for exclusion \\
\hline Kleis 2009 & $\begin{array}{l}\text { Wrong study design: compared FDG-PET to conventional imaging. No separate results for patients } \\
\text { with rhabdomyosarcoma }\end{array}$ \\
\hline Klem 2007 & Wrong study design: no FDG-PET/CT performed \\
\hline Kumar 2008 & $\begin{array}{l}\text { Wrong study design: compared FDG-PET to conventional imaging. No separate results for patients } \\
\text { with rhabdomyosarcoma }\end{array}$ \\
\hline Locantore 2013 & Wrong study design: compared FDG-PET to conventional imaging \\
\hline Ma 2015 & Conference proceeding, full report Dong 2017 \\
\hline Macpherson 2018 & Wrong study design: compared FDG-PET to conventional imaging \\
\hline Massardo 2012 & Only included 1 patient with rhabdomyosarcoma at time of diagnosis \\
\hline McCarville 2005 & No original research: review \\
\hline McCarville 2011 & Conference proceeding, full report included Federico 2013 \\
\hline Mody 2010 & Only included 1 patient with rhabdomyosarcoma \\
\hline Murphy 2008 & No original research: review \\
\hline Piperkova 2009 & Wrong study design: compared accuracy of PET and CT separately \\
\hline Reichert 2004 & No patients with rhabdomyosarcoma \\
\hline Ricard 2010 & Conference proceeding, full report included Ricard 2011 \\
\hline Sa 2020 & Wrong study design: no comparison described \\
\hline Sciuto 2014 & Study was not primary diagnostic. \\
\hline Sheikhbahaei 2015 & No original research: review \\
\hline Shin 2008 & No patients with rhabdomyosarcoma \\
\hline Singhal 2014 & Wrong study design: reference standard was bilateral bone marrow biopsy only. \\
\hline Sorschag 2011 & Study was not primary diagnostic. \\
\hline Tabacchi 2016 & No original research: review \\
\hline Tateishi 2007 & Partly same population as Tateishi 2009 \\
\hline Tateishi 2009 & $\begin{array}{l}\text { Wrong study design: compared FDG-PET to conventional imaging. Unclear how many patients with } \\
\text { rhabdomyosarcoma underwent FDG-PET/CT at staging. }\end{array}$ \\
\hline Terwisscha 2015 & Study was not primary diagnostic. \\
\hline Tezol 2020 & Wrong study design: reference standard was bilateral bone marrow biopsy only. \\
\hline Turpin 2016 & Study was not primary diagnostic. \\
\hline
\end{tabular}

Fluorine-18-fluorodeoxyglucose (FDG) positron emission tomography (PET) computed tomography (CT) for the detection of bone, lung, 


\begin{tabular}{ll}
\hline Study & Reason for exclusion \\
\hline Volker 2007 & Wrong study design: no FDG-PET/CT performed \\
\hline Wagner 2017 & Study was not primary diagnostic. \\
\hline Zapata 2015 & Conference proceeding, full report evaluated \\
\hline Zapata 2018 & Wrong study design: reference standard was bilateral bone marrow biopsy only. \\
\hline
\end{tabular}

Abbreviations: Cl: conventional imaging; CT: computed tomography; FDG-PET: fluorodeoxyglucose - positron emission tomography; PET/ $\mathrm{CT}$ : positron emission tomography/computed tomography; RMS: rhabdomyosarcoma.

\section{Characteristics of studies awaiting classification [ordered by study ID]}

\section{De Ferrater 2013}

\begin{tabular}{|c|c|}
\hline \multirow[t]{3}{*}{ Patient Sampling } & Inclusion period: June 2006 to December 2012 \\
\hline & Patient population: paediatric patients with head-neck malignancies excluding lymphoma \\
\hline & $\begin{array}{l}\text { Consecutive or random sample: consecutive patients who received a } 18 \mathrm{~F}-\mathrm{FDG}-\mathrm{PET} / \mathrm{CT} \text { at diagnosis, } \\
\text { during therapy, or at end of therapy }\end{array}$ \\
\hline
\end{tabular}

\begin{tabular}{|c|c|}
\hline \multirow{6}{*}{$\begin{array}{l}\text { Patient characteristics and } \\
\text { setting }\end{array}$} & Retrospective cohort study \\
\hline & A total of 31 patients were included: \\
\hline & $-\mathrm{RMS} n=9$ \\
\hline & Diagnostic workup: conventional imaging (CT, MRI, ultrasound, bone scan) \\
\hline & 161 scans were performed: 21 during staging, 42 during therapy, and 98 at end of treatment. \\
\hline & Number of patients with RMS and 18F-FDG-PET/CT at diagnosis was not reported. \\
\hline \multirow[t]{2}{*}{ Index tests } & 18F-FDG-PET/CT; information on tracer not reported, imaging protocol not reported \\
\hline & Information on interpreter and positive lesions of 18F-FDG-PET/CT not provided. \\
\hline \multirow{2}{*}{$\begin{array}{l}\text { Target condition and refer- } \\
\text { ence standard(s) }\end{array}$} & Target condition: paediatric patients with head-neck malignancies excluding lymphoma \\
\hline & Reference standard: histopathology or clinical follow-up or both, not further specified \\
\hline \multirow[t]{2}{*}{ Flow and timing } & Time between index test and reference standard not described. \\
\hline & Treatment between index test and reference standard not described. \\
\hline Comparative & $\begin{array}{l}\text { 18F-FDG-PET/CT had higher sensitivity and specificity compared to conventional imaging in staging } \\
\text { at initial diagnosis, not further specified for RMS only. }\end{array}$ \\
\hline \multirow[t]{4}{*}{ Notes } & As of July 2019 , this study has not been reported in full text. \\
\hline & No separate results for patients with RMS available. \\
\hline & Unclear if this study used histology or multidisciplinary tumour board as reference standard \\
\hline & We could not contact study authors via: mariaboronat@gmail.com. \\
\hline
\end{tabular}

Fluorine-18-fluorodeoxyglucose (FDG) positron emission tomography (PET) computed tomography (CT) for the detection of bone, lung, 
Mazurek 2011

\begin{tabular}{|c|c|}
\hline Patient Sampling & $\begin{array}{l}\text { Inclusion period: not described } \\
\text { Patient population: children with various types of sarcomas } \\
22 \text { patients included. } \\
\text { Consecutive or random sample: unclear }\end{array}$ \\
\hline $\begin{array}{l}\text { Patient characteristics and } \\
\text { setting }\end{array}$ & $\begin{array}{l}\text { Cohort study, not reported whether study was prospective or retrospective } \\
\text { A total of } 22 \text { patients were included: } \\
\text { - RMS, } n=6 \\
\text { Diagnostic workup: not reported } \\
22 \text { patients underwent } 18 \text { F-FDG-PET/CT for staging at diagnosis. }\end{array}$ \\
\hline Index tests & $\begin{array}{l}\text { 18F-FDG-PET/CT; images were acquired using a } 16 \text {-row PET-scanner, using } 0.21 \mathrm{mCi} / \mathrm{kg} 18 \mathrm{~F}-\mathrm{FDG} \\
\text { Images acquired } 60 \text { minutes after tracer injection. } \\
\text { Area of interest not reported. } \\
\text { Information on interpreter and positive lesions not reported. }\end{array}$ \\
\hline $\begin{array}{l}\text { Target condition and refer- } \\
\text { ence standard(s) }\end{array}$ & $\begin{array}{l}\text { Target condition: children with various types of sarcomas } \\
18 \text { F-FDG-PET/CT findings were compared with other imaging studies and with histopathology if } \\
\text { available. }\end{array}$ \\
\hline Flow and timing & $\begin{array}{l}\text { Time between index test and reference standard not described. } \\
\text { Treatment between index test and reference standard not described. }\end{array}$ \\
\hline Comparative & $\begin{array}{l}\text { Sensitivity or specificity not described. } \\
\text { No separate results reported for RMS. }\end{array}$ \\
\hline Notes & $\begin{array}{l}\text { As of July } 2019 \text {, this study has not been reported in full text. } \\
\text { No separate results for patients with RMS available. } \\
\text { Unclear if this study used histology or multidisciplinary tumour board as reference standard } \\
\text { Contact information for study authors not available. }\end{array}$ \\
\hline
\end{tabular}

\section{Nguyen 2011}

48 patients included.

Consecutive or random sample: consecutive

$\begin{array}{ll}\text { Patient characteristics and } & \text { Retrospective cohort study } \\ \text { setting } & 48 \text { patients included: }\end{array}$

Fluorine-18-fluorodeoxyglucose (FDG) positron emission tomography (PET) computed tomography (CT) for the detection of bone, lung, 


$$
\text { - RMS, } n=14
$$

Diagnostic workup: all included patients underwent an ${ }^{18} \mathrm{~F}-\mathrm{FDG}-\mathrm{PET} / \mathrm{CT}$ and ${ }^{99} \mathrm{Tc}$ bone scintigraphy, other diagnostic workup not reported

48 patients underwent 18 F-FDG-PET/CT.

\begin{tabular}{ll}
\hline Index tests & 18F-FDG-PET/CT; information on tracer not reported, imaging protocol not reported \\
& Information on interpreter and positive lesions of 18F-FDG-PET/CT not provided. \\
\hline $\begin{array}{l}\text { Target condition and refer- } \\
\text { ence standard(s) }\end{array}$ & $\begin{array}{l}\text { Target condition: patients with various types of sarcoma, } \\
\text { 18F-FDG-PET/CT findings were compared with }{ }^{99} \text { Tc bone scintigraphy, no gold standard described }\end{array}$ \\
\hline Flow and timing & Time between index test and reference standard: within 3 months \\
& Treatment between index test and reference standard not described. \\
\hline Comparative & No separate results reported for RMS. \\
\hline Notes & As of July 2019, this study has not been reported in full text. \\
No separate results for patients with RMS available. \\
Unclear if this study used histology or multidisciplinary tumour board as reference standard \\
Contact information for study authors not available.
\end{tabular}

\section{Oguz 2013}

Inclusion period: 1991 to 2013
Patient Sampling
18F-FDG-PET/CT at diagnosis
73 patients were included.
Consecutive or random sample: consecutive

\begin{tabular}{ll}
\hline Patient characteristics and & Retrospective cohort study \\
setting & 73 patients included: \\
& - soft-tissue sarcoma, $\mathrm{n}=8$ \\
& 58 patients underwent $18 \mathrm{~F}-\mathrm{FDG}-\mathrm{PET} / \mathrm{CT}$ for staging at initial diagnosis.
\end{tabular}

Index tests 18F-FDG-PET/CT information on tracer not reported, imaging protocol not reported.

Information on interpreter and positive lesions of 18 F-FDG-PET/CT not provided.

\begin{tabular}{ll}
\hline $\begin{array}{l}\text { Target condition and refer- } \\
\text { ence standard(s) }\end{array}$ & Target condition: patients with solid tumours outside central nervous system \\
& Reference standard not reported.
\end{tabular}

Flow and timing Time between index test and reference standard: not reported

Treatment between index test and reference standard not described. 
Oguz 2013 (Continued)
Comparative
No separate results reported for RMS.

Notes

As of July 2019, this study has not been reported in full text.

No separate results for patients with RMS available.

Unclear how many RMS patients were included, if any

Unclear if this study used histology or multidisciplinary tumour board as reference standard

Contact information for study authors not available.

\section{Riad 2010}

Inclusion period: not reported
Patient population: paediatric patients with histologically proven head and neck cancer
Consecutive or random sample: consecutive

\begin{tabular}{ll}
\hline $\begin{array}{l}\text { Patient characteristics and } \\
\text { setting }\end{array}$ & Retrospective cohort study \\
& 36 patients included: \\
& - RMS, $\mathrm{n}=9$ \\
& 9 patients underwent 18 F-FDG-PET/CT for staging at initial diagnosis, unclear if these patients had \\
RMS.
\end{tabular}

\begin{tabular}{ll}
\hline Index tests & 18F-FDG-PET/CT information on tracer not reported, imaging protocol not reported. \\
& 18F-FDG-PET/CT images were reviewed by 3 nuclear medicine specialists. \\
& Information on positive lesions of 18F-FDG-PET/CT not provided. \\
\hline $\begin{array}{l}\text { Target condition and refer- } \\
\text { ence standard(s) }\end{array}$ & Target condition: patients with histologically proven head and neck cancer \\
\hline Flow and timing & Reference standard: not reported \\
\hline Time between index test and reference standard: not reported \\
Treatment between index test and reference standard not described. \\
\hline Notes separate results reported for RMS. \\
As of July 2019, this study has not been reported in full text. \\
No separate results for patients with RMS available. \\
Unclear how many RMS patients underwent 18F-FDG-PET/CT at diagnosis, if any \\
Unclear if this study used histology or multidisciplinary tumour board as reference standard \\
Contact information for study authors not available. \\
\hline
\end{tabular}

Fluorine-18-fluorodeoxyglucose (FDG) positron emission tomography (PET) computed tomography (CT) for the detection of bone, lung, 
Sourabh 2010

Patient Sampling
Patient population: patients with bone and soft-tissue sarcoma
Consecutive or random sample: consecutive

\section{Patient characteristics and} setting

Retrospective cohort study

47 patients with bone and soft-tissue sarcoma were included: histological subtype not specified.

14 patients underwent a $18 \mathrm{~F}-\mathrm{FDG}-\mathrm{PET} / \mathrm{CT}$ for staging at initial diagnosis.

Index tests

18F-FDG-PET/CT information on tracer not reported, imaging protocol not reported.

18F-FDG-PET/CT images were reviewed by 3 nuclear medicine specialists.

Information on positive lesions of 18 F-FDG-PET/CT not provided.
Target condition and reference standard(s)

\section{Target condition: patients with histologically proven head and neck cancer}

Reference standard: not reported
Time between index test and reference standard: not reported

Treatment between index test and reference standard not described.

Comparative No separate results reported for RMS.

Notes

As of July 2019, this study has not been reported in full text.

No separate results for patients with RMS available.

Unclear how many RMS patients underwent 18 F-FDG-PET/CT at diagnosis, if any

Unclear if this study used histology or multidisciplinary tumour board as reference standard

Contact information for study authors not available.

\section{Tuncel 2015}

Patient Sampling
Patient population: paediatric patients with soft-tissue sarcoma
23 patients were included.
Consecutive or random sample: consecutive

Patient characteristics and

Cohort study, not reported whether retrospective or prospective

setting

23 patients included:

- RMS, $n=17$

Diagnostic workup: not reported

9 patients underwent a 18 F-FDG-PET/CT for staging. 
Tuncel 2015 (Continued)

Information on interpreter and positive lesions of $18 \mathrm{~F}-\mathrm{FDG}-\mathrm{PET} / \mathrm{CT}$ not provided.

\begin{tabular}{ll}
\hline $\begin{array}{l}\text { Target condition and refer- } \\
\text { ence standard(s) }\end{array}$ & $\begin{array}{l}\text { Target condition: paediatric patients with soft-tissue sarcoma } \\
\text { Reference standard: not reported }\end{array}$ \\
\hline Flow and timing & Time between index test and reference standard: not reported \\
& Treatment between index test and reference standard not described. \\
\hline Comparative & No separate data for 18F-FDG-PET/CT at initial diagnosis in RMS reported. \\
\hline Notes & As of July 2019, this study has not been reported in full text. \\
& No separate results for patients with RMS available. \\
Unclear how many RMS patients underwent 18F-FDG-PET/CT at diagnosis \\
Unclear if this study used histology or multidisciplinary tumour board as reference standard \\
Contact information for study authors not available.
\end{tabular}

Walter 2012

Patient Sampling
Patient population: paediatric patients with sarcoma
29 patients included.
Consecutive or random sample: consecutive

Patient characteristics and setting
Retrospective cohort study evaluating the diagnostic accuracy of $99 \mathrm{mTc}$ bone scintigraphy, ${ }^{18} \mathrm{~F}-$ FDG-PET/CT, and the combination for the assessment of bone involvement 29 patients included:

$$
\text { - RMS, } n=4
$$

Diagnostic workup: 99mTc bone scintigraphy, 18 F-FDG-PET/CT

10 patients underwent 18 F-FDG-PET/CT for staging.

Index tests $\quad$ 18F-FDG-PET/CT was acquired using $0.15 \mathrm{mCi} / \mathrm{kg}$ of $18 \mathrm{~F}-\mathrm{FDG}$.

Images were acquired 60 minutes after tracer injection.

Area of interest: whole body

18F-FDG-PET/CT images were reviewed by 1 nuclear medicine specialist and 1 paediatric radiologist.

Positive lesions: readers graded it as benign, likely benign, equivocal, likely malignant, or malignant

\begin{tabular}{ll}
\hline $\begin{array}{l}\text { Target condition and refer- } \\
\text { ence standard(s) }\end{array}$ & Target condition: paediatric patients with sarcoma \\
& $\begin{array}{l}\text { Reference standard: defined by the follow-up results including clinical, imaging results, and/or } \\
\text { biopsy, discussed in multidisciplinary tumour board. }\end{array}$
\end{tabular}

Flow and timing

Time between index test and reference standard: median 4 days \pm 7 days 
Walter 2012 (Continued)

Treatment between index test and reference standard: not reported

Comparative $\quad$ No separate data for 18 F-FDG-PET/CT at initial diagnosis in RMS reported.

Notes No separate results for patients with RMS available.

Unclear how many RMS patients underwent 18 F-FDG-PET/CT at diagnosis

Unclear if this study used histology or multidisciplinary tumour board as reference standard

We could not contact study authors via nfederman@mednet.ucla.edu.

Abbreviations: 18F-FDG-PET/CT: fluorine-18-fluorodeoxyglucose - positron emission tomography/computed tomography; 99mTc bone scintigraphy: technetium-99m bone scintigraphy; CT: computed tomography; $\mathrm{mCi} / \mathrm{kg}$ : millicurie per kilogram body weight; MRI: magnetic resonance imaging; RMS: rhabdomyosarcoma.

\section{A T A}

Presented below are all the data for all of the tests entered into the review.

Table Tests. Data tables by test

\begin{tabular}{lll}
\hline Test & No. of studies & No. of participants \\
\hline 1 18F-FDG-PET/CT for detection of bone metastases & 2 & 36 \\
\hline $2^{18 \text { F-FDG PET/CT for detection of lung metastases }}$ & 2 & 36 \\
\hline $3^{18 \text { F-FDG PET/CT for detection of nodal involvement }}$ & 2 & 36 \\
\hline
\end{tabular}

Test 1. 18F-FDG-PET/CT for detection of bone metastases

${ }^{18}$ F-FDG-PET/CT for detection of bone metastases

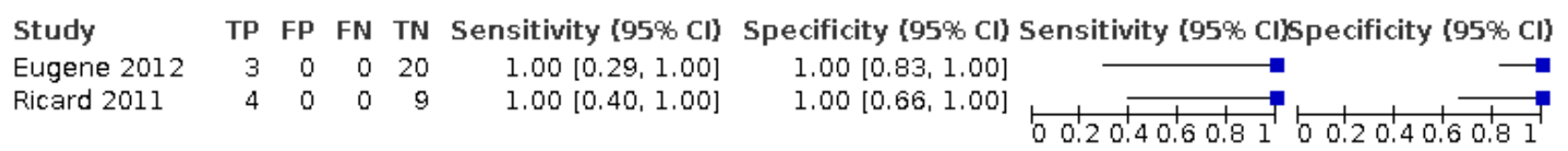

Test 2. 18F-FDG PET/CT for detection of lung metastases

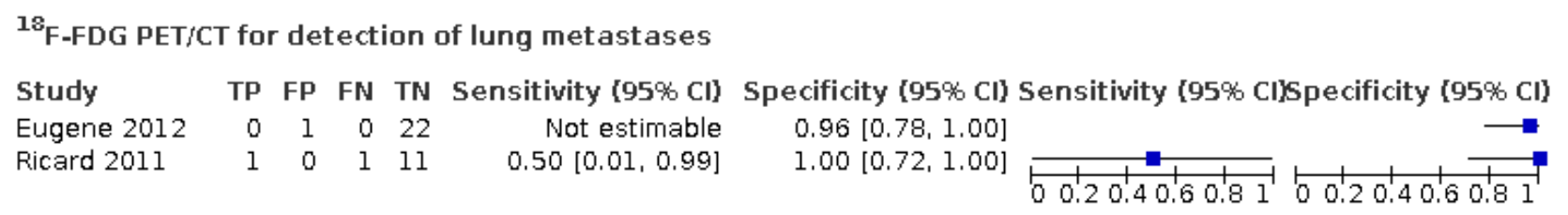




\title{
Test 3. 18F-FDG PET/CT for detection of nodal involvement
}

\author{
${ }^{13}$ F-FDG PET/CT for detection of nodal involvement
}

$\begin{array}{lrrrrrr}\text { Study } & \text { TP } & \text { FP } & \text { FN } & \text { TN } & \text { Sensitivity }(95 \% \mathrm{Cl}) & \text { Specificity }(95 \% \mathrm{Cl}) \text { Sensitivity }(95 \% \mathrm{Cl} \text { Specificity }(95 \% \mathrm{Cl}) \\ \text { Eugene } 2012 & 8 & 0 & 0 & 15 & 1.00[0.63,1.00] & 1.00[0.78,1.00] \\ \text { Ricard } 2011 & 4 & 1 & 0 & 8 & 1.00[0.40,1.00] & 0.89[0.52,1.00]\end{array}$

\section{ADDITIONAL TABLES}

Table 1. Items of the adapted QUADAS-2 tool and risk of bias and level of concerns about applicability

\section{Domain 1: Participant selection}

Was a consecutive or random sample of participants enrolled?
'Yes' if a consecutive or random sample of participants was enrolled

'No' if enrolled participants did not form a consecutive or random series

'Unclear' if the study did not describe the method of participant's enrolment
'Yes' if the study did not use a case-control design

'No' if the study used a case-control design

'Unclear' if the study reported insufficient information to ascertain whether a case-control design was used

Did the study describe exclusion criteria and were inappropriate exclusions avoided?

'Yes' if the characteristics of the participants were well described and inappropriate exclusions were avoided

'No' if participants were included that meet the exclusion criteria or inappropriate exclusions were not avoided

'Unclear' if the source or characteristics of participants was not adequately described

Low risk if 'yes' to all signalling questions

High risk if 'no' to any of the signalling questions

Unclear risk if there was insufficient information to judge the risk of bias

Is there concern that the included participants and setting do not match the review question?
A judgement oflow, high, or unclear concerns about applicability will be based on whether the exclusion criteria were well described and appropriate and how closely the sample matches the target population of interest.

Low concern if answer was 'yes' on the third signalling question and study population matched the target population

High concern if answer was 'no' on the third signalling question and the study population did not match the target population

Unclear concern if there was insufficient information to judge

\section{Domain 2: Index test ( 18 F-FDG-PET/CT)}

Were the results of the 'Yes' if the report stated that the person undertaking the index test did not know the results of the refer-
18F-FDG-PET/CT inter-
ence test
preted without knowl- 
Table 1. Items of the adapted QUADAS-2 tool and risk of bias and level of concerns about applicability (Continued) edge of the results of 'No' if the report stated that the same person performed both tests, or the results of the reference tests the reference standard? were known to the person undertaking the index tests

'Unclear' if insufficient information was provided

\section{If an SUV or lesion size 'Yes' if prespecified}

threshold was used,

was it prespecified?

'No' if not prespecified or the authors selected the optimal cut-off value based on the results of the study

'Unclear' if there was a range of cut-off values, and there was doubt as to which cut-off was used, or if no cut-off value was mentioned in the report

Did the study provide a clear definition of what was considered to be a positive test result?
'Yes' if the definition of a positive result was clearly stated (e.g. SUV)

'No' if no definition of what was considered to be a positive result was stated, or the definition of a positive result varied between participants

'Unclear' if insufficient information was provided to make a judgement
Were uninterpretable/intermediate test results reported?
'Yes' if it was clear that all information on uninterpretable and intermediate results was reported

'No' if uninterpretable results occurred but were not reported in detail

'Unclear' if it was not clear whether all test results were reported

\section{Could the conduct or interpretation of the $18 \mathrm{~F}$ - FDG-PET/CT have introduced bias?}

Low risk if 'yes' to all signalling questions

High risk if 'no' to any of the signalling questions

Unclear risk if there was insufficient information to judge the risk of bias
Are there concerns that the 18F-FDG-PET/CT, its conduct, or its interpretation differs from the review question?
Low concern if 'yes' to all signalling questions

High concern if the definition of a positive test result was not clear formulated, or if more than 1 signalling question was answered 'no'

Unclear concern if there was insufficient data to judge

\section{Domain 3: Reference standard}

Is the reference standard likely to correctly identify distant metastasis?

'Yes' if the correct conventional imaging modality was used (e.g. CT thorax for lung metastases, whole-body $99 \mathrm{mTc}$ skeleton scintigraphy for bone metastases, and MRI/ultrasound for nodal involvement) in combination with histological confirmation or confirmation by a tumour board opinion

'No' if the conventional imaging modality was not supported by histological confirmation or confirmation by a tumour board opinion.

'Unclear' if it was not clearly reported what reference standard was used

Were the results of the reference standard interpreted with blinding of the results of the $18 \mathrm{~F}-\mathrm{FDG}$ $\mathrm{PET} / \mathrm{CT}$ ?
'Yes' if the report stated that the person who was interpreting the reference test results did not know the results of the $18 \mathrm{~F}$-FDG-PET/CT

'No' if the report stated that the 18 F-FDG-PET/CT results were known to the person who was interpreting the reference tests results

'Unclear' if it was not reported whether blinding of the test results took place

\section{Could the reference standard, its conduct, or its interpretation have introduced bias?}

Low risk if 'yes' to all signalling questions

High risk if 'no' to any of the signalling questions 
Table 1. Items of the adapted QUADAS-2 tool and risk of bias and level of concerns about applicability (Continued) Unclear risk if there was insufficient information to judge the risk of bias

Is there concern that the target condition as defined by the reference standard does not match the review question?
Low concern for identification of the primary tumour as the reference standard defined, this will always be confirmed by histopathology. Histological confirmation will not always be available for lymph node involvement and distant metastases.

High concern when the study does not report how false-negative and falsepositive results were obtained

\section{Domain 4: Flow and timing}

Did all participants receive the same reference standard?
'Yes' if the same reference test was used in all included participants regardless of the index test results

'No' if different reference tests were used to verify the disease status depending on the results of the index test

'Unclear' if there was insufficient information to determine whether different reference standards were used

Were all participants included in the analysis?
'Yes' if no were participants excluded from the analysis, or if exclusions were adequately described

'No' if participants were excluded from the analysis, and no explanation was given

'Unclear' if there was insufficient information to determine whether all participants were included in the analysis
Was the delay between the performance of the $18 \mathrm{~F}$ FDG-PET/CT and the reference standard less than two weeks?
'Yes' if the period between 18 F-FDG-PET/CT and the reference standard was less than two weeks, and no treatment was started

'No' if the period between $18 \mathrm{~F}$-FDG-PET/CT and the reference standard was more than two weeks, or treatment was already started

'Unclear' if there was insufficient information about the time period between tests

\section{Could the participant flow have introduced bias? Low risk if 'yes' to all signalling questions}

High risk if 'no' to any of the signalling questions

Unclear risk if there was insufficient information to judge the risk of bias

18F-FDG-PET/CT: fluorine-18-fluorodeoxyglucose - positron emission tomography/computed tomography; CT: computed tomography; MRI: magnetic resonance imaging; SUV: standardised uptake value.

Table 2. Summary of participant characteristics

\begin{tabular}{lll}
\hline & Eugene 2012 (n= 23) & Ricard 2011 (n= 13) \\
\hline Median age at diagnosis & 8.7 & 9.6 \\
(years) & range: 0.8 to 21.6 & range: 1.8 to 19.1 \\
\hline Sex & $16(70 \%)$ & $12(92 \%)$ \\
Male & $7(30 \%)$ & $1(8 \%)$ \\
\hline
\end{tabular}

Fluorine-18-fluorodeoxyglucose (FDG) positron emission tomography (PET) computed tomography (CT) for the detection of bone, lung, 
Table 2. Summary of participant characteristics (Continued)

Female

\begin{tabular}{|c|c|c|}
\hline Histology & $13(57 \%)$ & $3(23 \%)$ \\
\hline Embryonal & $9(39 \%)$ & $10(77 \%)$ \\
\hline Alveolar & - & - \\
\hline Spindle cell & $1(4 \%)$ & - \\
\hline Botryoid RMS & - & - \\
\hline Mixed histology & - & - \\
\hline \multicolumn{3}{|l|}{ RMS NOS } \\
\hline Primary tumour site & $5(22 \%)$ & - \\
\hline Orbit & $2(9 \%)$ & $4(31 \%)$ \\
\hline Head-neck non-parameningeal & $5(22 \%)$ & $2(15 \%)$ \\
\hline Parameningeal & $4(17 \%)$ & $3(23 \%)$ \\
\hline GU - bladder/prostate & - & - \\
\hline GU - non-bladder/prostate & $5(22 \%)$ & $4(31 \%)$ \\
\hline Extremity & $1(4 \%)$ & - \\
\hline Other & $1(4 \%)$ & - \\
\hline \multicolumn{3}{|l|}{ Primary site unknown } \\
\hline Postsurgical staging* & Not reported & $4(31 \%)$ \\
\hline IRS & & $1(8 \%)$ \\
\hline IRS II & & $2(15 \%)$ \\
\hline IRS III & & $6(46 \%)$ \\
\hline IRS IV & & \\
\hline
\end{tabular}

${ }^{*} \mathrm{IRS} I$ = primary complete resection (R0); IRS II = microscopic residual (R1) or primary complete resection but N1; IRS III = macroscopic residual (R2); IRS IV = distant metastatic disease present at onset.

Abbreviations: GU: genito-urinary; NOS: not otherwise specified; RMS: rhabdomyosarcoma.

\section{AP PE N D I CES}

\section{Appendix 1. Search strategy for MEDLINE/PubMed}

1. For rhabdomyosarcoma, we used the following MeSH headings and text words:

rhabdomyosarcom* OR rhabdomyosarcoma OR rhabdomyosarcomas OR embryonal rhabdomyosarcom* OR embryonal rhabdomyosarcoma OR embryonal rhabdomyosarcomas OR rhabdomyosarcomas, embryonal OR alveolar rhabdomyosarcom* OR alveolar rhabdomyosarcoma OR alveolar rhabdomyosarcomas OR rhabdomyosarcomas, alveolar OR myosarcom* OR myosarcoma OR myosarcomas OR soft tissue sarcom^ OR soft tissue sarcoma[tiab] OR soft tissue sarcomas[tiab] OR botryoid sarcoma[tiab]

2. For 18-F-FDG-PET/CT scan, we used the following MeSH headings and text words:

Fluorine-18-fluorodeoxyglucose (FDG) positron emission tomography (PET) computed tomography (CT) for the detection of bone, lung, 38 and lymph node metastases in rhabdomyosarcoma (Review)

Copyright (C) 2021 The Cochrane Collaboration. Published by John Wiley \& Sons, Ltd. 
Positron Emission Tomography[mh] OR Positron Emission Tomography[tiab] OR Positron Emission Tomograph* OR PET Scan OR PET Scans OR PET Scan* OR PET OR SPECT OR SPECT-CT OR tomography, emission-computed, single-photon[mh] OR Single Photon Emission Computed Tomography[tiab] OR Single photon emission computerized tomography[tiab] OR Single photon emission computerised tomography[tiab] OR Single Photon Emission Computed Radionuclide Tomography[tiab] OR Single Photon Emission CT Scan[tiab] OR Single Photon Emission CAT scan[tiab] OR Single Photon Emission Computer Assisted Tomography[tiab] OR Single Photon Emission Computed Radionuclide Tomograph* OR Single Photon Emission CT Scan* OR Single Photon Emission CAT scan* OR Single Photon Emission Computer Assisted Tomograph ${ }^{\star}$ OR Single Photon Emission Computed Tomograph* OR Single photon emission computerized tomograph* OR Single photon emission computerised tomograph* OR 18F-FDG-PET-CT OR 18 F-FDG-PET OR 18-fluorodeoxy* OR 18fluorodeoxy ${ }^{\star}$ OR fdgpet OR fdg pet OR $18 \mathrm{ffdg} \mathrm{fOR}^{\star}$ fluorodeoxyglucose $\mathrm{f} 18$

Final search (1 AND 2) NOT (case reports OR case report)

* $=$ zero or more characters

\section{Appendix 2. Search strategy for Embase (Ovid)}

1. For rhabdomyosarcoma, we used the following Emtree terms and text words:

1. Rhabdomyosarcoma/ or embryonal rhabdomyosarcoma/

2. Soft Tissue Sarcoma/ or myosarcoma/

3. (myosarcom\$ or myosarcoma or myosarcomas or soft tissue sarcom\$ or soft tissue sarcoma or soft tissue sarcomas).mp.

4. (rhabdomyosarcom\$ or rhabdomyosarcoma or rhabdomyosarcomas).mp.

5. (embryonal rhabdomyosarcom\$ or embryonal rhabdomyosarcoma or embryonal rhabdomyosarcomas or embryo rhabdomyosarcoma).mp.

6. (alveolar rhabdomyosarcom\$ or alveolar rhabdomyosarcoma or alveolar rhabdomyosarcomas or alveolus-like rhabdomyosarcoma).mp.

7. botryoid sarcoma.mp.

8. or/1-7

2. For 18F-FDG-PET/CT scan, we used the following Emtree terms and text words:

1. exp positron emission tomography/ or exp fluorodeoxyglucose $\mathrm{f18/}$

2. (positron emission tomography or positron emission tomograph\$).mp.

3. (PET scan or PET scans or PET scan\$ or PET).mp.

4. (SPECT or SPECT-CT or 18F-FDG-PET-CT).mp.

5. exp single photon emission computer tomography/

6. (single photon emission computed tomography or single photon emission computed tomograph\$ or single photon emission computerized tomography or single photon emission computerised tomography).mp.

7. (Single photon emission computerized tomograph\$ or Single photon emission computerised tomograph\$).mp.

8. (single photon emission computed radionuclide tomography or single photon emission computed radionuclide tomograph\$).mp.

9. (Single Photon Emission CT Scan or Single Photon Emission CT Scan\$).mp.

10. (Single Photon Emission CAT scan or Single Photon Emission CAT scan\$).mp.

11. (Single Photon Emission Computer Assisted Tomography or Single Photon Emission Computer Assisted Tomograph\$).mp.

12. (18 F-FDG-PET or 18-fluorodeoxy\$ or 18fluorodeoxy $\$$ or $\mathrm{fdg}$ pet or fdgpet or $18 \mathrm{ffdg}$ or $18 \mathrm{ffdg}$ or fluorodeoxyglucose $\mathrm{f18}$ ).mp.

13. or/1-12

Final search was (1 AND 2) NOT (case reports OR case report)

$\mathrm{mp}=$ title, abstract, subject headings, heading word, drug trade name, original title, device manufacturer, drug manufacturer name; / = Emtree term; \$=zero or more characters

\section{HISTORY}

Protocol first published: Issue 8, 2016

\section{CONTRIBUTIONS OFAUTHORS}

BV: reviewed the draft protocol and performed study selection and data extraction, QUADAS-2 assessment, set up data and analysis of tables; created additional tables; wrote to authors of papers for additional information; drafted the manuscript.

WB: designed and drafted the protocol, performed the study selection and data extraction, QUADAS- 2 assessment, and revised the manuscript.

LH: reviewed the draft protocol and revised the manuscript.

Fluorine-18-fluorodeoxyglucose (FDG) positron emission tomography (PET) computed tomography (CT) for the detection of bone, lung, 
JFC: reviewed draft protocol, provided methodological support, helped with data analysis and revised the manuscript for important intellectual content.

EvD, BdK, RvR, HvdB: drafted the protocol, interpreted data and revised the manuscript, gave general advice on the review.

NJ, LB: interpreted data and revised the manuscript, gave general advice on the review.

JM: drafted the protocol and manuscript; managed the review process, third-party arbitrator for study selection, data extraction, and QUADAS-2 assessment.

\section{DECLARATIONS OF INTEREST}

JHM: None known

BV: None known

WBB: None known

LMH: None known

BdK: None known

$\mathrm{NJ}$ : None known

LB: None known

RRvR: None known

HvdB: None known

JFC: None known

ECvD: None known

\section{SOURCES OF SUPPORT}

\section{Internal sources}

- No sources of support provided

\section{External sources}

- Tom Voûte Fund, Netherlands

- Stichting Kinderen Kankervrij (KiKa), Netherlands

\section{DIFFERENCES BETWEEN PROTOCOL AND REVIEW}

The protocol can be found here.

\section{Regarding the reference standard}

We clarified the definition of lung metastases by changing the sentence "In case of doubt or several small (less than 5 mm) nodules, a multidisciplinary tumour board decides whether a biopsy was indicated to confirm the diagnosis." to "In case of doubt or 5 or more small (less than $5 \mathrm{~mm}$ ) nodules, a multidisciplinary tumour board decides whether a biopsy was indicated to confirm the diagnosis." This definition is also used in the EpSSG RMS 2005 protocol (Bisogno 2018).

\section{Regarding the analyses}

We intended to perform formal meta-analysis with meta-regression and sensitivity analyses, but the studies were too heterogeneous and data were limited, so we considered this not useful.

Due to the limited data we did not obtain receiver operating characteristic (ROC) plots, and we did not provide likelihood ratios estimates.

\section{N DEX TERMS}

\section{Medical Subject Headings (MeSH)}

Cross-Sectional Studies; *Fluorodeoxyglucose F18; Lung; Lymphatic Metastasis; Neoplasm Staging; Positron Emission Tomography Computed Tomography; Positron-Emission Tomography; Prospective Studies; Radiopharmaceuticals; Retrospective Studies; ${ }^{\star}$ Rhabdomyosarcoma [diagnostic imaging]; Sensitivity and Specificity; Tomography, X-Ray Computed

\section{MeSH check words}

Humans

Fluorine-18-fluorodeoxyglucose (FDG) positron emission tomography (PET) computed tomography (CT) for the detection of bone, lung, 